\title{
Open-Ended Metallodithiolene Complexes with the 1,2,4,5- Tetrakis(diphenylphosphino)benzene Ligand: Modular Building Elements for the Synthesis of Multimetal Complexes
}

Satyendra Kumar,* Malathy Selvachandran, Kuppuswamy Arumugam, Mohamed C. Shaw, Che Wu, Michael Maurer, Xiaodong Zhang, Stephen Sproules, Joel T. Mague, and James P. Donahue*

Cite This: Inorg. Chem. 2021, 60, 13177-13192

Read Online

ABSTRACT: Open-ended, singly metalated dithiolene complexes with 1,2,4,5-tetrakis(diphenylphosphino)benzene (tpbz) are prepared either by ligand transfer to $\left[\mathrm{Cl}_{2} \mathrm{M}(\mathrm{tpbz})\right]$ from $\left(\mathrm{R}_{2} \mathrm{C}_{2} \mathrm{~S}_{2}\right) \mathrm{SnR}_{2}{ }_{2}(\mathrm{R}=$ $\left.\mathrm{CN}, \mathrm{R}^{\prime}=\mathrm{Me} ; \mathrm{R}=\mathrm{Me}, \mathrm{R}^{\prime}={ }^{n} \mathrm{Bu}\right)$ or by a direct reaction between tpbz and $\left[\mathrm{M}\left(\mathrm{S}_{2} \mathrm{C}_{2} \mathrm{R}_{2}\right)_{2}\right](\mathrm{M}=\mathrm{Ni}, \mathrm{Pd}, \mathrm{Pt} ; \mathrm{R}=\mathrm{Ph}, p$-anisyl $)$ in a $1: 1$ ratio. The formation of dimetallic $\left[\left(\mathrm{R}_{2} \mathrm{C}_{2} \mathrm{~S}_{2}\right) \mathrm{M}(\mathrm{tpbz}) \mathrm{M}\left(\mathrm{S}_{2} \mathrm{C}_{2} \mathrm{R}_{2}\right)\right]$ attends these syntheses in modest amounts, but the open-ended compounds are readily separated by silica chromatography. As affirmed by X-ray crystallographic characterization of numerous members of the set, the $\left[\left(\mathrm{R}_{2} \mathrm{C}_{2} \mathrm{~S}_{2}\right) \mathrm{M}(\mathrm{tpbz})\right]$ compounds show dithiolene ligands in their fully reduced ene-1,2-dithiolate form conjoined with divalent Group 10 ions. Minor amounts of octahedral $\left[\left(\mathrm{Ph}_{2} \mathrm{C}_{2} \mathrm{~S}_{2}\right)_{2} \mathrm{Pt}^{\mathrm{IV}}(\mathrm{tpbz})\right]$, a

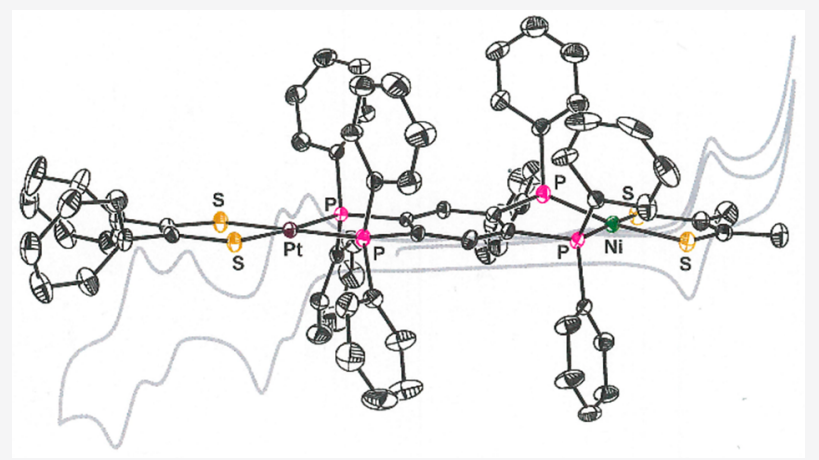
presumed intermediate, are isolated from the preparation of $\left[\left(\mathrm{Ph}_{2} \mathrm{C}_{2} \mathrm{~S}_{2}\right) \mathrm{Pt}^{\mathrm{II}}(\mathrm{tpbz})\right]$. Heterodimetallic $\left[\left(\mathrm{Ph}_{2} \mathrm{C}_{2} \mathrm{~S}_{2}\right) \mathrm{Pt}(\mathrm{tpbz}) \mathrm{Ni}\left(\mathrm{S}_{2} \mathrm{C}_{2} \mathrm{Me}_{2}\right)\right]$ is prepared from $\left[\left(\mathrm{Ph}_{2} \mathrm{C}_{2} \mathrm{~S}_{2}\right) \mathrm{Pt}^{\mathrm{II}}(\mathrm{tpbz})\right]$; its cyclic voltammogram, upon anodic scanning, shows two pairs of closely spaced, but resolved, $1 \mathrm{e}^{-}$oxidations corresponding first to $\left[\mathrm{R}_{2} \mathrm{C}_{2} \mathrm{~S}_{2}{ }^{2-}\right]-1 \mathrm{e}^{-} \rightarrow\left[\mathrm{R}_{2} \mathrm{C}_{2} \mathrm{~S}^{\bullet} \mathrm{S}^{-}\right]$and then to $\left[\mathrm{R}_{2} \mathrm{C}_{2} \mathrm{~S}^{\bullet} \mathrm{S}^{-}\right]-1 \mathrm{e}^{-} \rightarrow\left[\mathrm{R}_{2}(\mathrm{C}=\mathrm{S})_{2}\right]$. The open diphosphine of $\left[\left(\mathrm{R}_{2} \mathrm{C}_{2} \mathrm{~S}_{2}\right) \mathrm{M}(\mathrm{tpbz})\right]$ can be oxidized to afford open-ended $\left[\left(\mathrm{R}_{2} \mathrm{C}_{2} \mathrm{~S}_{2}\right) \mathrm{M}\left(\mathrm{tpbzE}_{2}\right)\right](\mathrm{E}=\mathrm{O}, \mathrm{S})$. Synthesis of the octahedral $\left[\left(\mathrm{dppbO}_{2}\right)_{3} \mathrm{Ni}_{[}\right]\left[\mathrm{I}_{3}\right]_{2}\left[\mathrm{dppbO}_{2}=1,2-\right.$ bis(diphenylphosphoryl)benzene $]$ suggests that the steric profile of $\left[\left(\mathrm{R}_{2} \mathrm{C}_{2} \mathrm{~S}_{2}\right) \mathrm{M}\left(\mathrm{tpbzE}_{2}\right)\right]$ is moderated enough that three could be accommodated as ligands around a metal ion.

\section{INTRODUCTION}

In recent work, ${ }^{1,2}$ we have reported the synthesis, structures, and properties of a set of dimetallic compounds of the type $\left[\left(\mathrm{R}_{2} \mathrm{C}_{2} \mathrm{~S}_{2}\right) \mathrm{M}(\mathrm{tpbz}) \mathrm{M}\left(\mathrm{S}_{2} \mathrm{C}_{2} \mathrm{R}_{2}\right)\right] \quad[\mathrm{tpbz}=1,2,4,5$-tetrakis(diphenylphosphino)benzene], where $\mathrm{R}$ may be $\mathrm{CN}, \mathrm{Me}, \mathrm{Ph}$, or $p$-anisyl and, independent of $\mathrm{R}, \mathrm{M}$ may be varied as $\mathrm{Ni}^{2+}$, $\mathrm{Pd}^{2+}$, or $\mathrm{Pt}^{2+}$. The dithiolene end groups can be concurrently oxidized to radical monoanions $(\mathbf{a} \rightarrow \mathbf{b}$, Scheme 1), thus providing $\left[\left(\mathrm{R}_{2} \mathrm{C}_{2} \mathrm{~S}^{-} \mathrm{S}^{\bullet}\right) \mathrm{M}(\mathrm{tpbz}) \mathrm{M}\left(\mathrm{S}^{-} \mathrm{S}^{\bullet} \mathrm{C}_{2} \mathrm{R}_{2}\right)\right]^{2+}$ dications, which weakly couple to provide nearly isoenergetic $S=1$ and 0 states in equilibrium. Electron paramagnetic resonance (EPR) spectroscopy is effective in characterizing subtle

Scheme 1. Redox Levels Available to a Dithiolene Ligand When Bound to a Transition Metal

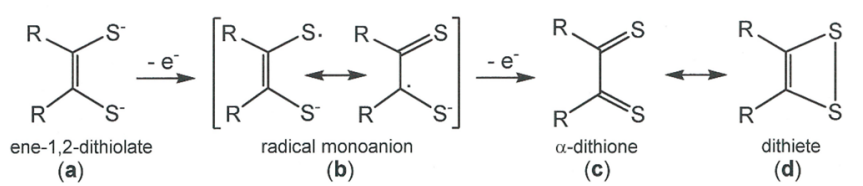

differences among the $\left[\left(\mathrm{R}_{2} \mathrm{C}_{2} \mathrm{~S}_{2}\right) \mathrm{M}(\mathrm{tpbz}) \mathrm{M}\left(\mathrm{S}_{2} \mathrm{C}_{2} \mathrm{R}_{2}\right)\right]^{2+}$ complexes as $\mathrm{M}$ and $\mathrm{R}$ are varied. For example, simulations of the EPR spectra of $\left[\left(\left(\mathrm{MeO}-p-\mathrm{C}_{6} \mathrm{H}_{4}\right){ }_{2} \mathrm{C}_{2} \mathrm{~S}_{2}\right) \mathrm{M}(\mathrm{tpbz}) \mathrm{M}\right.$ $\left.\left(\mathrm{S}_{2} \mathrm{C}_{2}\left(\mathrm{C}_{6} \mathrm{H}_{4}-\mathrm{p}-\mathrm{OMe}\right)_{2}\right)\right]^{2+}$, where $\mathrm{M}=\mathrm{Ni}^{2+}$ or $\mathrm{Pd}^{2+}$, yield $\mathrm{D}$ $=-18 \times 10^{-4} \mathrm{~cm}^{-1}$ and $-15 \times 10^{-4} \mathrm{~cm}^{-1}$, respectively, with the former value indicative of a slightly shorter distance between the spin barycenters because of greater spin delocalization onto the metal from the dithiolene radicals.

The conditions for the syntheses of $\left[\left(\mathrm{R}_{2} \mathrm{C}_{2} \mathrm{~S}_{2}\right) \mathrm{M}(\mathrm{tpbz}) \mathrm{M}\right.$ $\left.\left(\mathrm{S}_{2} \mathrm{C}_{2} \mathrm{R}_{2}\right)\right]$ that we reported were devised to promote formation of the dimetallic species. However, when the tpbz ligand is introduced to a source of the Group 10 metal bis(dithiolene) complex in an amount that is greater than the $1: 2$ ratio that is optimal for $\left[\left(\mathrm{R}_{2} \mathrm{C}_{2} \mathrm{~S}_{2}\right) \mathrm{M}(\mathrm{tpbz}) \mathrm{M}\left(\mathrm{S}_{2} \mathrm{C}_{2} \mathrm{R}_{2}\right)\right]$, we

Received: May 25, 2021

Published: August 9, 2021 


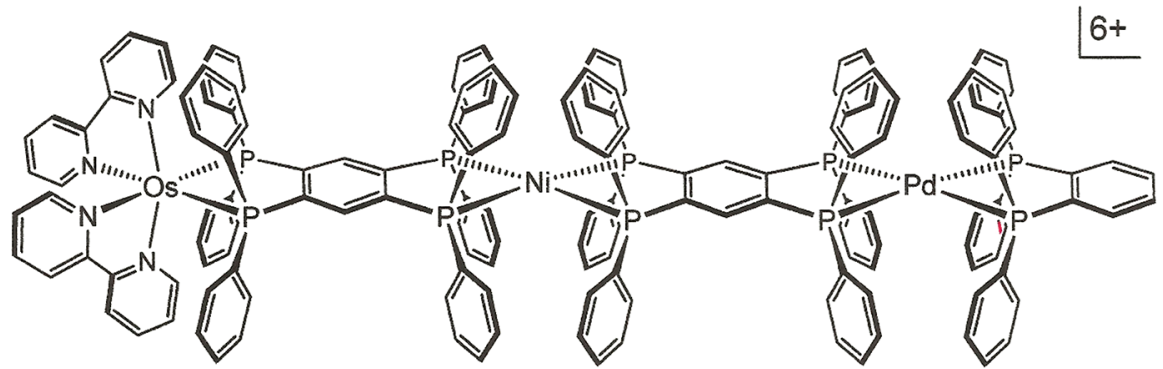

Figure 1. A previously reported ${ }^{3}$ tpbz-linked heterotrimetallic complex designed to support gated photoinduced electron transfer.

Scheme 2. Syntheses of Open-Ended $\left[\left(\mathrm{R}_{2} \mathrm{C}_{2} \mathrm{~S}_{2}\right) \mathrm{M}(\mathrm{tpbz})\right]\left(\mathrm{M}=\mathrm{Ni}^{2+}, \mathrm{Pd}^{2+}, \mathrm{Pt}^{2+} ; \mathrm{R}=\mathrm{Me}, \mathrm{Ph}, \mathrm{CH}_{3} \mathrm{O}-\mathrm{p}-\mathrm{C}_{6} \mathrm{H}_{4}, \mathrm{CN}\right) \mathrm{Compounds}$
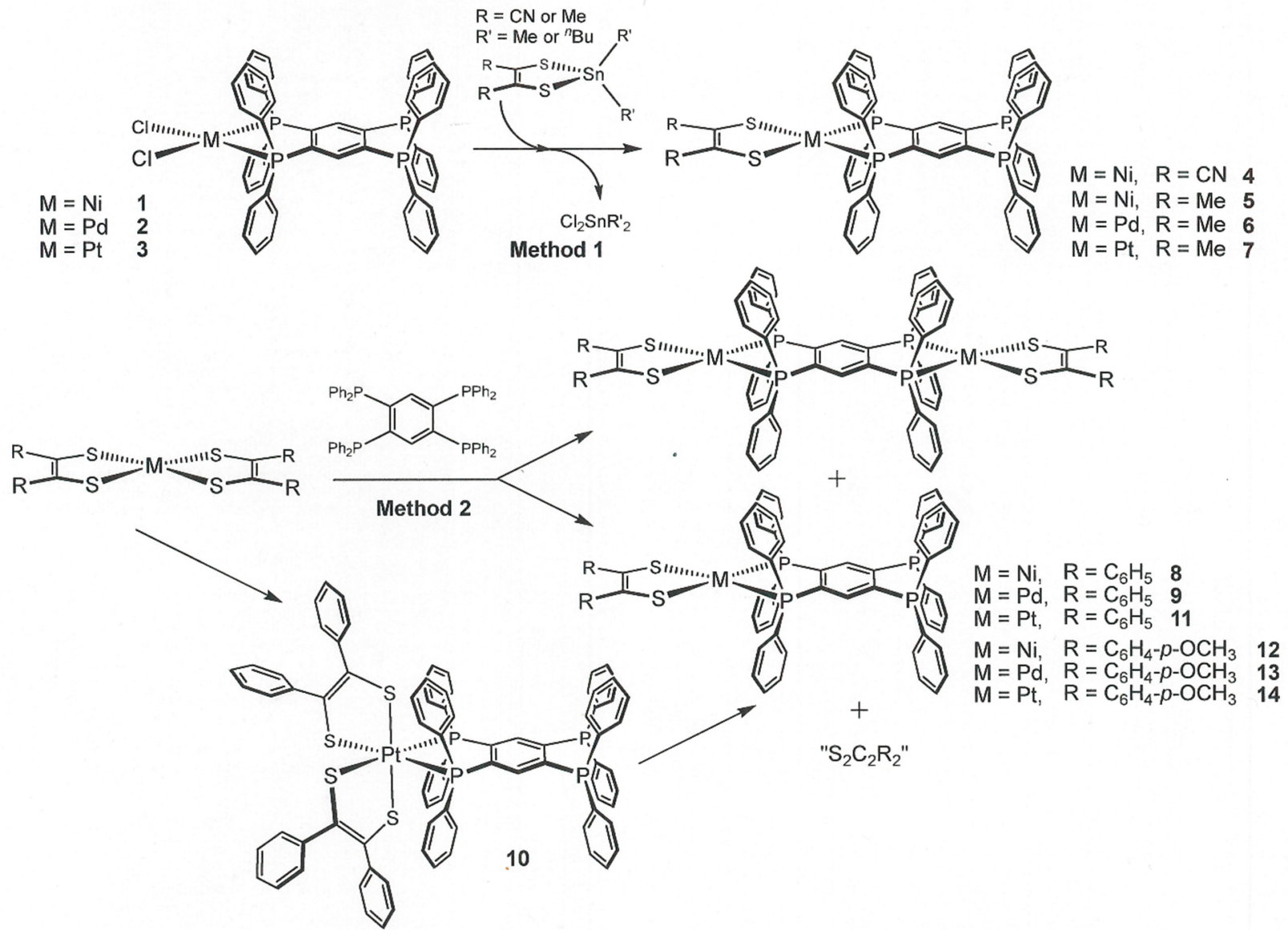

have noted that "open-ended" tpbz compounds can be isolated in which only one of the two chelating sites is occupied. Although the centrosymmetic dimetal compounds still form, they are readily separated from the open-ended compounds because of their lowered solubility and slower movement on silica columns. These open-ended monometallic compounds still possess the air and moisture stability enjoyed by the free tpbz ligand itself, thus making them easily accessible in quantities that can be deployed for further synthetic ends. In every sense of the term, these open-ended complexes are themselves ligands, or "modules," for the systematic creation of higher-order assemblies incorporating other metal ions with the capacity for a specific function. Zahavy and Fox, for example, described trimetallic [(bpy $)_{2} \mathrm{Os}(\mathrm{tpbz}) \mathrm{Ni}(\mathrm{tpbz}) \mathrm{Pd}$ $(\mathrm{dppb})]^{6+}$ (Figure 1), the synthesis of which proceeded through open-ended $\left[(\mathrm{bpy})_{2} \mathrm{Os}(\mathrm{tpbz})\right]^{2+}$, as a photoinduced
Os $\rightarrow$ Pd electron transfer device with gating controlled by the redox state at $\mathrm{Ni}^{3}$

In general terms, modular chemical synthesis may be described as the preparation of materials or discrete compounds from two or more sets of parts such that (1) an array of products whose number is the product of the numbers of the members of the constituents sets can be obtained, (2) a common synthetic methodology can be executed for the synthesis of all products, and (3) desirable properties of the resulting materials, such as the redox potential or acidity, may be varied with a predictable effect. Arguably, the most prominent example of modular synthesis is the synthesis of metal-organic frameworks, wherein "nodes" defined by transition-metal ions with varying coordination environment constraints are joined by any of an array of chemical linkers to produce open spacings or channels. ${ }^{4}$ A rather different example from $\mathrm{Lu}$ and co-workers is the installment, via a two-step 
Scheme 3. Syntheses of Pt-Ni Heterodimetallic 15 and Oxidized Open-Ended Compounds 16-18

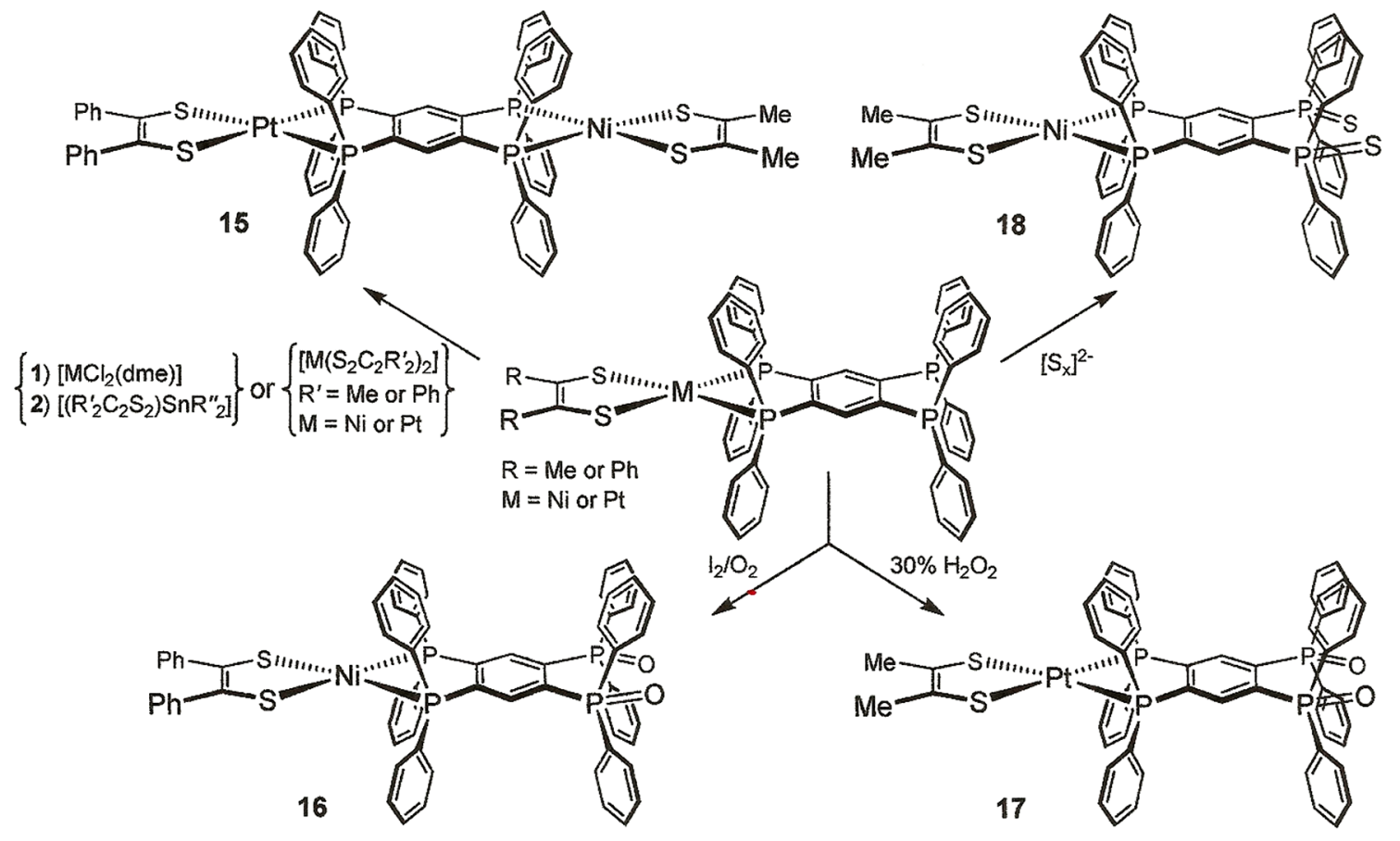

metalation protocol, of heterodimetallic units within a variety of tripodal ligands whose arms feature three-atom bridges. ${ }^{5}$ An obvious advantage of modular synthesis is the efficiency it can provide toward identifying an optimal candidate for a particular application.

In this report, we provide an account of the synthesis and characterization of the open-ended $\left[\left(\mathrm{R}_{2} \mathrm{C}_{2} \mathrm{~S}_{2}\right) \mathrm{M}(\mathrm{tpbz})\right]$ compounds that are summarized pictorially in Scheme 2. A particular emphasis is placed on the solid-state molecular structures and on ${ }^{31} \mathrm{P}$ NMR spectroscopy as a convenient and definitive handle for characterization of these compounds. Following their isolation, postsynthetic modification of $\left[\left(\mathrm{R}_{2} \mathrm{C}_{2} \mathrm{~S}_{2}\right) \mathrm{M}(\mathrm{tpbz})\right]$ by oxidation of the noncoordinating $\mathrm{P}$ sites to the corresponding phosphine oxide or sulfide is possible and provides a further dimension of variability for this metallodithiolene "module" (Scheme 3). Using isolated $\left[\left(\mathrm{R}_{2} \mathrm{C}_{2} \mathrm{~S}_{2}\right) \mathrm{M}(\mathrm{tpbz})\right]$, asymmetric heterodimetallic complexes of the form $\left[\left(\mathrm{R}_{2} \mathrm{C}_{2} \mathrm{~S}_{2}\right) \mathrm{M}(\mathrm{tpbz}) \mathrm{M}^{\prime}\left(\mathrm{S}_{2} \mathrm{C}_{2} \mathrm{R}_{2}^{\prime}\right)\right]\left(\mathrm{M}^{\prime} \neq \mathrm{M} ; \mathrm{R} \neq\right.$ $\mathrm{R}^{\prime}$ ) are readily prepared that are not accessible by the method originally disclosed for $\left[\left(\mathrm{R}_{2} \mathrm{C}_{2} \mathrm{~S}_{2}\right) \mathrm{M}(\mathrm{tpbz}) \mathrm{M}\left(\mathrm{S}_{2} \mathrm{C}_{2} \mathrm{R}_{2}\right)\right]$.

\section{SYNTHESES}

General Considerations. Literature methods were implemented for the syntheses of $\left[\mathrm{Cl}_{2} \mathrm{Ni}(\mathrm{dme})\right]^{6}(\mathrm{dme}=1,2$-dimethoxyethane $)$, $\left[\mathrm{Cl}_{2} \mathrm{M}\left(\mathrm{NCCH}_{3}\right)_{2}\right]\left(\mathrm{M}=\mathrm{Pd}^{2+}, \mathrm{Pt}^{2+}\right),{ }^{7}\left[\left(\mathrm{R}_{2} \mathrm{C}_{2} \mathrm{~S}_{2}\right)_{2} \mathrm{M}\right](\mathrm{R}=\mathrm{Me}, \mathrm{Ph}, p-$ anisyl; $\left.\mathrm{M}=\mathrm{Ni}^{2+}, \mathrm{Pd}^{2+}, \mathrm{Pt}^{2+}\right),{ }^{8}\left[\left((\mathrm{NC})_{2} \mathrm{C}_{2} \mathrm{~S}_{2}\right) \mathrm{SnMe}_{2}\right],\left[\left(\mathrm{Me}_{2} \mathrm{C}_{2} \mathrm{~S}_{2}\right)-\right.$ $\left.\mathrm{Sn}\left({ }^{n} \mathrm{Bu}\right)_{2}\right]$, the tpbz ligand, ${ }^{9}$ and 1,2-bis(diphenylphosphino)benzene dioxide $\left(\mathrm{dppbO}_{2}\right) \cdot{ }^{10}$ All other reagents were purchased from commercial sources and used as received. Solvents were either dried with a system of drying columns from the Glass Contour Company [dichloromethane $\left(\mathrm{CH}_{2} \mathrm{Cl}_{2}\right)$, n-pentane, hexanes, diethyl ether $\left(\mathrm{Et}_{2} \mathrm{O}\right)$, tetrahydrofuran $(\mathrm{THF})$, benzene $\left(\mathrm{C}_{6} \mathrm{H}_{6}\right)$, and toluene] or freshly distilled according to standard procedures [methanol $(\mathrm{MeOH})$, acetonitrile $\left(\mathrm{CH}_{3} \mathrm{CN}\right)$, and 1,2-dichloroethane $]{ }^{11}$ All reactions described below were conducted under an atmosphere of $\mathrm{N}_{2}$, while silica columns were run in the open air using $60-230 \mu \mathrm{m}$ silica (Dynamic Adsorbents). Dithiolene ligand abbreviations used throughout the text are as follows: mdt $=\left[\mathrm{Me}_{2} \mathrm{C}_{2} \mathrm{~S}_{2}\right]^{2-}=1,2-$ dimethyl-1,2-dithiolate $(2-) ; \mathrm{mnt}=\left[(\mathrm{NC})_{2} \mathrm{C}_{2} \mathrm{~S}_{2}\right]^{2-}=$ maleonitriledithiolate(2-); pdt $=\left[\mathrm{Ph}_{2} \mathrm{C}_{2} \mathrm{~S}_{2}\right]^{2-}=1,2$-diphenyl-1,2-dithiolate(2-); adt $=\left[\left(\mathrm{MeO}-p-\mathrm{C}_{6} \mathrm{H}_{4}\right)_{2} \mathrm{C}_{2} \mathrm{~S}_{2}\right]^{2-}=1,2$-di- $p$-anisyl-1,2-dithiolate $(2-)$.

Physical Methods. All ${ }^{1} \mathrm{H}$ and ${ }^{31} \mathrm{P}$ NMR spectra were recorded at $25{ }^{\circ} \mathrm{C}$ with a Bruker Avance spectrometer operating at 300.13 and $121.49 \mathrm{MHz}$ for the ${ }^{1} \mathrm{H}$ and ${ }^{31} \mathrm{P}$ nuclei, respectively. The ${ }^{1} \mathrm{H}$ NMR spectra were referenced to the solvent signal, while an external aqueous phosphoric acid $\left(\mathrm{H}_{3} \mathrm{PO}_{4}\right)$ solution was employed as the reference for all ${ }^{31} \mathrm{P}$ NMR spectra. Mass spectra (positive-ion electrospray ionization, $\mathrm{ESI}^{+}$) were obtained with a Bruker micrOTOF II mass spectrometer. The X-band EPR spectrum was recorded on a Bruker ELEXSYS E500 spectrometer. Electrochemical measurements were made with a CHI620C electroanalyzer workstation using a $\mathrm{Ag} / \mathrm{AgCl}$ reference electrode, a glassy carbon or Pt disk working electrode, a Pt wire auxiliary electrode, and a $\left[{ }^{n} \mathrm{Bu}_{4} \mathrm{~N}\right]\left[\mathrm{PF}_{6}\right]$ supporting electrolyte. Under these conditions, the $\mathrm{Cp}_{2} \mathrm{Fe}^{+} / \mathrm{Cp}_{2} \mathrm{Fe}$ $\left(\mathrm{Fc}^{+} / \mathrm{Fc}\right)$ couple consistently occurred at $+0.436 \mathrm{mV}$ in $\mathrm{CH}_{2} \mathrm{Cl}_{2}$. Elemental analyses were performed by Midwest Microlab, LLC (Indianapolis, IN), Galbraith Laboratories, Inc. (Knoxville, TN), or Kolbe Microanalytical Laboratory (Oberhausen, Germany). Procedural details regarding crystal growth, $\mathrm{X}$-ray diffraction data collection, data processing, and structure solution and refinement are available in the Supporting Information. Unit cell data and selected refinement statistics are presented in Table 1.

$\left[\mathrm{Cl}_{2} \mathrm{Ni}(\mathrm{tpbz})\right]$ (1). A $50 \mathrm{~mL}$ Schlenk flask charged with $\left[\mathrm{Cl}_{2} \mathrm{Ni}-\right.$ (dme)] (0.022 g, $0.10 \mathrm{mmol})$, tpbz $(0.081 \mathrm{~g}, 0.10 \mathrm{mmol})$, and $\mathrm{CH}_{2} \mathrm{Cl}_{2}(15 \mathrm{~mL})$ was stirred for $1 \mathrm{~h}$ at ambient temperature, during which time a dark-red color developed. The reaction mixture was filtered to remove undissolved materials, and the filtrate was taken to dryness under reduced pressure to afford an orange-red solid residual that was suitable for use without further purification. Yield: $0.075 \mathrm{~g}$, $0.079 \mathrm{mmol}, 79 \%$. ${ }^{1} \mathrm{H}$ NMR $\left(\mathrm{CD}_{2} \mathrm{Cl}_{2}\right): \delta 9.63-9.51(\mathrm{~m}, 11 \mathrm{H}$, aromatic $\mathrm{C}-\mathrm{H}), 9.34-9.15(\mathrm{~m}, 14 \mathrm{H}$, aromatic $\mathrm{C}-\mathrm{H}), 8.98-8.78(\mathrm{~m}$, $17 \mathrm{H}$, aromatic $\mathrm{C}-\mathrm{H}) .{ }^{31} \mathrm{P}\left\{{ }^{1} \mathrm{H}\right\}$ NMR $\left(\mathrm{CD}_{2} \mathrm{Cl}_{2}\right): \delta 49.9$ (s), -14.1 (s).

$\left[\mathrm{Cl}_{2} \mathrm{Pd}(\mathrm{tpbz})\right]$ (2). A $50 \mathrm{~mL}$ Schlenk flask was charged with $\left[\mathrm{PdCl}_{2}\left(\mathrm{CH}_{3} \mathrm{CN}\right)_{2}\right](0.026 \mathrm{~g}, 0.10 \mathrm{mmol}), \mathrm{tpbz}(0.081 \mathrm{~g}, 0.10 \mathrm{mmol})$, and a 1:1 mixture of $\mathrm{CH}_{2} \mathrm{Cl}_{2} / \mathrm{CH}_{3} \mathrm{CN}(20 \mathrm{~mL})$. The resulting mixture was stirred at ambient temperature for $12 \mathrm{~h}$ in the dark, during which time a light-yellow solution developed. The solvent was removed under reduced pressure, and the residual light-yellow solid was 


\section{Table 1. Unit Cell and Refinement Data for Compounds Characterized by X-ray Diffraction}

\begin{tabular}{|c|c|c|c|c|}
\hline compound & {$\left[\mathrm{Cl}_{2} \mathrm{Ni}(\mathrm{tpbz})\right]$} & {$[(\mathrm{mnt}) \mathrm{Ni}(\mathrm{tpbz})]$} & {$[(\mathrm{mdt}) \mathrm{Ni}(\mathrm{tpbz})]$} & {$[(\mathrm{mdt}) \mathrm{Pt}(\mathrm{tpbz})]$} \\
\hline compound no. & 1 & 4 & 5 & 7 \\
\hline cocryst solvent & $\mathrm{Et}_{2} \mathrm{O}$ & $2 \mathrm{CHCl}_{3}$ & $2 \mathrm{CH}_{2} \mathrm{Cl}_{2}$ & $2 \mathrm{CH}_{2} \mathrm{Cl}_{2}$ \\
\hline formula & $\mathrm{C}_{58} \mathrm{H}_{52} \mathrm{Cl}_{2} \mathrm{NiOP}_{4}$ & $\mathrm{C}_{60} \mathrm{H}_{44} \mathrm{Cl}_{6} \mathrm{~N}_{2} \mathrm{NiP}_{4} \mathrm{~S}_{2}$ & $\mathrm{C}_{60} \mathrm{H}_{52} \mathrm{Cl}_{4} \mathrm{NiP}_{4} \mathrm{~S}_{2}$ & $\mathrm{C}_{60} \mathrm{H}_{52} \mathrm{Cl}_{4} \mathrm{P}_{4} \mathrm{PtS}_{2}$ \\
\hline fw, $\mathrm{g} \mathrm{mol}^{-1}$ & 1018.49 & 1252.38 & 1161.52 & 1297.91 \\
\hline temperature, $\mathrm{K}$ & 100 & 100 & 100 & 100 \\
\hline wavelength, Å & 0.71073 & 0.71073 & 0.71073 & 0.71073 \\
\hline $2 \theta$ range, deg & $2.34-55.70$ & $4.22-56.74$ & $3.24-60.44$ & $3.22-56.56$ \\
\hline cryst syst & orthorhombic & orthorhombic & monoclinic & monoclinic \\
\hline space group & $\operatorname{Pna2}_{1}$ & $P 2_{1} 2_{1} 2_{1}$ & $C 2 / c$ & $\mathrm{C} 2 / \mathrm{c}$ \\
\hline$a, \AA$ & $34.703(3)$ & $13.510(3)$ & $21.223(2)$ & $21.157(5)$ \\
\hline$b, \AA$ & $9.2939(9)$ & $16.403(3)$ & $15.7180(18)$ & $15.836(4)$ \\
\hline$c, \AA$ & $15.5064(14)$ & $25.380(5)$ & $16.7123(18)$ & $16.576(4)$ \\
\hline$\alpha, \operatorname{deg}$ & 90 & 90 & 90 & 90 \\
\hline$\beta, \operatorname{deg}$ & 90 & 90 & $100.039(2)$ & 99.604(4) \\
\hline$\gamma, \operatorname{deg}$ & 90 & 90 & 90 & 90 \\
\hline volume $\left(\AA^{3}\right), Z$ & $5001.2(8), 4$ & 5624.2(19), 4 & 5489.6(11), 4 & $5476(2), 4$ \\
\hline density, $\mathrm{g} \mathrm{cm}^{-3}$ & 1.353 & 1.479 & 1.405 & 1.574 \\
\hline$\mu, \mathrm{mm}^{-1}$ & 0.664 & 0.860 & 0.780 & 2.991 \\
\hline color, habit & orange plate & orange block & brwn-grn column & yellow plate \\
\hline limiting indices $h$ & $-44 \leq h \leq 45$ & $-17 \leq h \leq 18$ & $-29 \leq h \leq 29$ & $-28 \leq h \leq 27$ \\
\hline limiting indices $k$ & $-12 \leq k \leq 12$ & $-21 \leq k \leq 21$ & $-22 \leq k \leq 22$ & $-20 \leq k \leq 20$ \\
\hline limiting indices $l$ & $-20 \leq l \leq 19$ & $-33 \leq l \leq 33$ & $-23 \leq l \leq 23$ & $-21 \leq l \leq 21$ \\
\hline reflns collected & 40918 & 100119 & 52873 & 24025 \\
\hline indep data, param ${ }^{a}$ & 11569,597 & 14060,676 & 7799,322 & 6526,322 \\
\hline $\mathrm{GOF}^{b}$ & 1.090 & 1.048 & 1.105 & 1.035 \\
\hline $\mathrm{R} 1,^{c, d} \mathrm{wR}^{d, e}$ & $0.0574,0.1259$ & $0.0413,0.1087$ & $0.0450,0.1434$ & $0.0308,0.0750$ \\
\hline $\mathrm{R} 1,^{c_{1} f} \mathrm{wR}^{e_{2} f}$ & $0.0725,0.1327$ & $0.0481,0.1141$ & $0.0590,0.1468$ & $0.0360,0.0776$ \\
\hline compound & {$[(\mathrm{pdt}) \mathrm{Ni}(\mathrm{tpbz})]$} & {$[(\mathrm{pdt}) \operatorname{Pd}(\mathrm{tpbz})]$} & {$[(\mathrm{pdt}) \mathrm{Pt}(\mathrm{tpbz})]$} & {$\left[(\mathrm{pdt})_{2} \mathrm{Pt}(\mathrm{tpbz})\right]$} \\
\hline compound no. & 8 & 9 & 11 & 10 \\
\hline cocryst solvent & none & none & none & $2\left(\mathrm{ClCH}_{2} \mathrm{CH}_{2} \mathrm{Cl}\right)$ \\
\hline formula & $\mathrm{C}_{68} \mathrm{H}_{52} \mathrm{NiP}_{4} \mathrm{~S}_{2}$ & $\mathrm{C}_{68} \mathrm{H}_{52} \mathrm{P}_{4} \mathrm{PdS}_{2}$ & $\mathrm{C}_{68} \mathrm{H}_{52} \mathrm{P}_{4} \mathrm{PtS}_{2}$ & $\mathrm{C}_{86} \mathrm{H}_{70} \mathrm{Cl}_{4} \mathrm{P}_{4} \mathrm{PtS}_{4}$ \\
\hline $\mathrm{fw}, \mathrm{g} \mathrm{mol}^{-1}$ & 1115.80 & 1163.49 & 1252.18 & 1692.43 \\
\hline temperature, $\mathrm{K}$ & 150 & 100 & 100 & 100 \\
\hline wavelength, Å & 1.54178 & 0.71073 & 0.71073 & 0.71073 \\
\hline $2 \theta$ range, deg & $6.84-148.77$ & $3.14-58.44$ & $3.14-57.39$ & $3.03-59.72$ \\
\hline cryst syst & monoclinic & monoclinic & monoclinic & triclinic \\
\hline space group & $C 2 / c$ & $\mathrm{C} 2 / \mathrm{c}$ & $C 2 / c$ & $P \overline{1}$ \\
\hline a, $\AA$ & $21.9519(4)$ & $21.7574(15)$ & $21.7804(15)$ & $13.9324(12)$ \\
\hline$b, \AA$ & $16.7831(3)$ & $16.9716(12)$ & $16.9661(12)$ & $14.0989(12)$ \\
\hline$c, \AA$ & $16.4070(3)$ & $16.2670(11)$ & $16.2774(12)$ & $21.2092(18)$ \\
\hline$\alpha, \operatorname{deg}$ & 90 & 90 & 90 & $89.716(1)$ \\
\hline$\beta$, deg & $112.864(1)$ & $112.666(1)$ & $112.727(1)$ & $84.452(1)$ \\
\hline$\gamma, \operatorname{deg}$ & 90 & 90 & 90 & $72.605(1)$ \\
\hline volume $\left(\AA^{3}\right), Z$ & $5569.75(18), 4$ & $5542.8(7), 4$ & $5547.9(7), 4$ & $3955.7(6), 2$ \\
\hline density, $\mathrm{g} \mathrm{cm}^{-3}$ & 1.331 & 1.394 & 1.499 & 1.421 \\
\hline$\mu, \mathrm{mm}^{-1}$ & 2.612 & 0.569 & 2.763 & 2.139 \\
\hline color, habit & yellow block & pale-orange column & yellow column & dark-blue block \\
\hline limiting indices $h$ & $-27 \leq h \leq 27$ & $-29 \leq h \leq 28$ & $-29 \leq h \leq 28$ & $-19 \leq h \leq 19$ \\
\hline limiting indices $k$ & $-20 \leq k \leq 20$ & $-23 \leq k \leq 23$ & $-22 \leq k \leq 22$ & $-19 \leq k \leq 19$ \\
\hline limiting indices $l$ & $-20 \leq l \leq 20$ & $-22 \leq l \leq 22$ & $-21 \leq l \leq 21$ & $-29 \leq l \leq 29$ \\
\hline reflns collected & 35518 & 26293 & 26168 & 77174 \\
\hline indep data, param ${ }^{a}$ & 5650,339 & 7084,339 & 7003,339 & 21625,909 \\
\hline $\mathrm{GOF}^{b}$ & 1.063 & 1.074 & 1.006 & 1.071 \\
\hline $\mathrm{R} 1,^{c, d} \mathrm{wR}^{d, e}$ & $0.0340,0.0918$ & $0.0408,0.1122$ & $0.0362,0.0836$ & $0.0379,0.1019$ \\
\hline $\mathrm{R} 11^{c, f} \mathrm{wR}^{e, f}$ & $0.0376,0.0952$ & $0.0558,0.1179$ & $0.0487,0.0870$ & $0.0433,0.1044$ \\
\hline
\end{tabular}

${ }^{a}$ Independent data collected and parameters refined. ${ }^{b} \mathrm{GOF}=\left\{\sum\left[w\left(F_{\mathrm{o}}{ }^{2}-F_{\mathrm{c}}{ }^{2}\right)^{2}\right] /(n-p)\right\}^{1 / 2}$, where $n=$ number of reflections and $p$ is the total number of parameters refined. ${ }^{c} \mathrm{R} 1=\sum\left\|F_{\mathrm{o}}|-| F_{\mathrm{c}}\right\| / \sum\left|F_{\mathrm{o}}\right|{ }^{d} \mathrm{R}$ indices for data cut off at $I>2 \sigma(I) .{ }^{e} \mathrm{wR} 2=\left\{\sum\left[w\left(F_{\mathrm{o}}{ }^{2}-F_{\mathrm{c}}{ }^{2}\right)^{2}\right] / \sum\left[w\left(\mathrm{~F}_{\mathrm{o}}{ }^{2}\right)^{2}\right]\right\}^{1 / 2} ; w=$ $1 /\left[\sigma^{2}\left(F_{\mathrm{o}}{ }^{2}\right)+(x P)^{2}+y P\right]$, where $P=\left[2 F_{\mathrm{c}}{ }^{2}+\operatorname{Max}\left(F_{\mathrm{o}}{ }^{2}, 0\right)\right] / 3 .{ }^{f_{R}}$ indices for all data.

washed with $\mathrm{Et}_{2} \mathrm{O}(2 \times 5 \mathrm{~mL})$ and dried in vacuo. This compound shows only limited solubility in common organic solvents. Yield:
$0.084 \mathrm{~g}, 0.085 \mathrm{mmol}, 85 \% .{ }^{1} \mathrm{H}$ NMR (DMSO- $\left.d_{6}\right): \delta 7.63-7.52(\mathrm{~m}$, $14 \mathrm{H}$, aromatic $\mathrm{C}-\mathrm{H}), 7.35-7.16(\mathrm{~m}, 14 \mathrm{H}$ aromatic $\mathrm{C}-\mathrm{H})$, 6.94- 
$6.79(\mathrm{~m}, 14 \mathrm{H}$, aromatic $\mathrm{C}-H) .{ }^{31} \mathrm{P}\left\{{ }^{1} \mathrm{H}\right\}$ NMR (DMSO-d 6 ): $\delta 49.5$ (s), $-14.4(\mathrm{~s})$.

$\left[\mathrm{Cl}_{2} \mathrm{Pt}(\mathrm{tpbz})\right]$ (3). The same procedure and scale as those described for the synthesis of $\mathbf{2}$ were implemented using [ $\left.\mathrm{PtCl}_{2}\left(\mathrm{CH}_{3} \mathrm{CN}\right)_{2}\right]$, which yielded 3 as a white solid. Yield: $0.083 \mathrm{~g}$, $0.077 \mathrm{mmol}, 77 \%$. ${ }^{1} \mathrm{H}$ NMR (DMSO- $\left.d_{6}\right): \delta 7.81-7.74(\mathrm{~m}, 12 \mathrm{H}$, aromatic $\mathrm{C}-\mathrm{H}), 7.64-7.56(\mathrm{~m}, 22 \mathrm{H}$, aromatic $\mathrm{C}-\mathrm{H}), 7.30-7.22(\mathrm{~m}$, $8 \mathrm{H}$, aromatic $\mathrm{C}-H) .{ }^{31} \mathrm{P}\left\{{ }^{1} \mathrm{H}\right\}$ NMR (DMSO-d $d_{6}$ ): $\delta 39.1(\mathrm{~s}),-14.1$ (s).

[(mnt)Ni(tpbz)] (4). Under an atmosphere of $\mathrm{N}_{2}$, a $50 \mathrm{~mL}$ Schlenk flask with a stirbar was charged with $1(0.088 \mathrm{~g}, 0.093 \mathrm{mmol})$ and 20 $\mathrm{mL}$ of $\mathrm{CH}_{2} \mathrm{Cl}_{2}$. Under an outward flow of $\mathrm{N}_{2}$, solid [(mnt) $\left.\mathrm{SnMe}_{2}\right]$ $(0.029 \mathrm{~g}, 0.10 \mathrm{mmol})$ was added to the flask, and the resulting mixture was stirred at ambient temperature for $12 \mathrm{~h}$, during which time a reddish-brown color developed. The solvent was removed under reduced pressure, and the reddish crude solid was then triturated with $\mathrm{MeOH}(2 \times 5 \mathrm{~mL})$, followed by $\mathrm{Et}_{2} \mathrm{O}(2 \times 5 \mathrm{~mL})$, and dried under vacuum. This crude material was further purified on a silica column eluted with $\mathrm{CH}_{2} \mathrm{Cl}_{2}$ and collected as a red-brown band. Recrystallization was accomplished by the diffusion of $\mathrm{MeOH}$ into a filtered $\mathrm{CHCl}_{3}$ solution. Yield: $0.070 \mathrm{~g}, 74 \% . \mathrm{R}_{f}=0.17\left(9: 1 \mathrm{CH}_{2} \mathrm{Cl}_{2} /\right.$ hexanes $)$. ${ }^{1} \mathrm{H}$ NMR $\left(\mathrm{CDCl}_{3}\right): \delta 7.37-7.32(\mathrm{~m}, 4 \mathrm{H}$, aromatic $\mathrm{C}-\mathrm{H}), 7.22-7.12$ $(\mathrm{m}, 21 \mathrm{H}$, aromatic $\mathrm{C}-\mathrm{H}), 7.06-6.97(\mathrm{~m}, 9 \mathrm{H}$, aromatic $\mathrm{C}-\mathrm{H}), 6.90-$ $6.85(\mathrm{~m}, 8 \mathrm{H}$, aromatic $\mathrm{C}-\mathrm{H}) .{ }^{31} \mathrm{P}\left\{{ }^{1} \mathrm{H}\right\}$ NMR $\left(\mathrm{CDCl}_{3}\right): \delta 57.2(\mathrm{~s})$, -14.5 (s). UV-vis $\left[\mathrm{CH}_{2} \mathrm{Cl}_{2} ; \lambda_{\max }, \mathrm{nm}\left(\varepsilon, \mathrm{M}^{-1} \mathrm{~cm}^{-1}\right)\right]: 366$ (4960). IR $\left(\mathrm{KBr}, \mathrm{cm}^{-1}\right): 2217\left(\nu_{\mathrm{C}=\mathrm{N}}\right.$, symm $), 2204\left(\nu_{\mathrm{C} \equiv \mathrm{N}}\right.$, asymm $) . \mathrm{MS}$ $\left(\mathrm{ESI}^{+}\right)$. Calcd for monoisotopic $\left[\mathrm{C}_{58} \mathrm{H}_{42} \mathrm{~N}_{2} \mathrm{NiP}_{4} \mathrm{~S}_{2}\right]^{+}: m / z 1012.1093$. Obsd: $m / z$ 1012.1041. Error $(\delta): 5.2 \mathrm{ppm}$.

[(Me $\left.\left.\mathrm{C}_{2} \mathrm{~S}_{2}\right) \mathrm{Ni}(\mathrm{tpbz})\right]$ (5). A $50 \mathrm{~mL}$ Schlenk flask with a stirbar was charged with $1(0.088 \mathrm{~g}, 0.093 \mathrm{mmol})$ and $20 \mathrm{~mL}$ of $\mathrm{CH}_{2} \mathrm{Cl}_{2}$. Under an outward flow of $\mathrm{N}_{2}$, solid $\left[\left(\mathrm{Me}_{2} \mathrm{C}_{2} \mathrm{~S}_{2}\right) \mathrm{Sn}\left({ }^{n} \mathrm{Bu}\right)_{2}\right]$ (0.0351 g, 0.10 $\mathrm{mmol}$ ) was added to the flask, which immediately induced a darkgreen-brown color. The resulting mixture was stirred at ambient temperature for $12 \mathrm{~h}$. The solvent was removed under reduced pressure, and the solid residual was triturated with $\mathrm{MeOH}(2 \times 5$ $\mathrm{mL})$, followed by $\mathrm{Et}_{2} \mathrm{O}(2 \times 5 \mathrm{~mL})$, and then dried under vacuum. This material was purified on a silica chromatography column eluted with $\mathrm{CH}_{2} \mathrm{Cl}_{2} /$ hexanes (9:1) and collected as a green band. Recrystallization was accomplished by the diffusion of $n$-pentane or $\mathrm{Et}_{2} \mathrm{O}$ into a filtered $\mathrm{CH}_{2} \mathrm{Cl}_{2}$ solution. Yield: $0.044 \mathrm{~g}, 48 \% . R_{f}=0.32$ (9:1 $\mathrm{CH}_{2} \mathrm{Cl}_{2} /$ hexanes). ${ }^{1} \mathrm{H}$ NMR $\left(\mathrm{CDCl}_{3}\right): \delta 7.37-7.24$ (overlapping $\mathrm{m}, 12 \mathrm{H}$, aromatic $\mathrm{C}-\mathrm{H}), 7.15-7.10(\mathrm{~m}, 12 \mathrm{H}$, aromatic $\mathrm{C}-\mathrm{H}), 7.03-$ $6.95(\mathrm{~m}, 10 \mathrm{H}$, aromatic $\mathrm{C}-\mathrm{H}), 6.88-6.84(\mathrm{~m}, 8 \mathrm{H}$, aromatic $\mathrm{C}-\mathrm{H})$, $2.00\left(\mathrm{~s}, 6 \mathrm{H},-\mathrm{CH}_{3}\right) .{ }^{31} \mathrm{P}\left\{{ }^{1} \mathrm{H}\right\}$ NMR $\left(\mathrm{CDCl}_{3}\right): \delta 55.1(\mathrm{~s}),-14.9(\mathrm{~s})$. UV-vis $\left[\mathrm{CH}_{2} \mathrm{Cl}_{2} ; \lambda_{\max }, \mathrm{nm}\left(\varepsilon, \mathrm{M}^{-1} \mathrm{~cm}^{-1}\right)\right]: 444$ (1440), 638 (160). MS $\left(\mathrm{ESI}^{+}\right)$. Calcd for monoisotopic $\left[\mathrm{C}_{58} \mathrm{H}_{48} \mathrm{NiP}_{4} \mathrm{~S}_{2}\right]^{+}: m / z$ 990.1501. Obsd: $m / z$ 990.1509. Error $(\delta): 0.76 \mathrm{ppm}$.

$\left[\left(\mathrm{Me}_{2} \mathrm{C}_{2} \mathrm{~S}_{2}\right) \mathrm{Pd}(\mathrm{tpbz})\right]$ (6). The same procedure and scale as those described for the synthesis of $\mathbf{4}$ were implemented but with $\mathbf{2}$ used in place of the corresponding $\mathrm{Ni}$ compound. Purification was accomplished by a chromatography column eluted with $\mathrm{CH}_{2} \mathrm{Cl}_{2}$ / hexanes (3:1), with 5 collected as a yellow band. Yield: $0.032 \mathrm{~g}, 33 \%$. $R_{f}=0.20$ (9:1 $\mathrm{CH}_{2} \mathrm{Cl}_{2} /$ hexanes). ${ }^{1} \mathrm{H} \mathrm{NMR}\left(\mathrm{CDCl}_{3}\right): \delta 7.35-7.25(\mathrm{~m}$, $12 \mathrm{H}$, aromatic $\mathrm{C}-\mathrm{H}), 7.18-7.12(\mathrm{~m}, 14 \mathrm{H}$, aromatic $\mathrm{C}-\mathrm{H}), 7.06-$ $7.01(\mathrm{~m}, 8 \mathrm{H}$, aromatic $\mathrm{C}-\mathrm{H}), 6.89-6.84(\mathrm{~m}, 8 \mathrm{H}$, aromatic $\mathrm{C}-\mathrm{H})$, $2.01\left(\mathrm{~s}, 6 \mathrm{H},-\mathrm{CH}_{3}\right) .{ }^{31} \mathrm{P}\left\{{ }^{1} \mathrm{H}\right\} \mathrm{NMR}\left(\mathrm{CDCl}_{3}\right): \delta 49.3(\mathrm{~s}),-14.7(\mathrm{~s})$. $\mathrm{UV}-$ vis $\left[\mathrm{CH}_{2} \mathrm{Cl}_{2} ; \lambda_{\max } \mathrm{nm}\left(\varepsilon, \mathrm{M}^{-1} \mathrm{~cm}^{-1}\right)\right]: 427$ (1450), 588 (360). MS $\left(\mathrm{ESI}^{+}\right)$. Calcd for monoisotopic $\left[\mathrm{C}_{58} \mathrm{H}_{48} \mathrm{P}_{4} \mathrm{PdS}_{2}\right]^{+}: \mathrm{m} / z$ 1038.1201. Obsd: $m / z$ 1038.1159. Error $(\delta): 4.11 \mathrm{ppm}$.

$\left[\left(\mathrm{Me}_{2} \mathrm{C}_{2} \mathrm{~S}_{2}\right) \mathrm{Pt}(\mathrm{tpbz})\right]$ (7). The same procedure and scale as those described for the synthesis of $\mathbf{4}$ were implemented but with 3 used in place of the corresponding $\mathrm{Ni}$ compound. Purification was accomplished using a silica column chromatography eluted with $\mathrm{CH}_{2} \mathrm{Cl}_{2}$ /hexanes (2:1); 6 was collected as a yellow band. Yield: 0.041 g, 39\%. $R_{f}=0.35$ (9:1 $\mathrm{CH}_{2} \mathrm{Cl}_{2} /$ hexanes). ${ }^{1} \mathrm{H}$ NMR $\left(\mathrm{CDCl}_{3}\right): \delta 7.39-$ $7.33(\mathrm{~m}, 8 \mathrm{H}$, aromatic $\mathrm{C}-\mathrm{H}), 7.29-7.24(\mathrm{~m}, 4 \mathrm{H}$, aromatic $\mathrm{C}-\mathrm{H})$, $7.19-7.13(\mathrm{~m}, 14 \mathrm{H}$, aromatic $\mathrm{C}-\mathrm{H}), 7.06-7.01(\mathrm{~m}, 8 \mathrm{H}$, aromatic $\mathrm{C}-\mathrm{H}), 6.90-6.85(\mathrm{~m}, 8 \mathrm{H}$, aromatic $\mathrm{C}-\mathrm{H}), 2.07\left(\mathrm{~s}, 6 \mathrm{H},-\mathrm{CH}_{3}\right)$. ${ }^{31} \mathrm{P}\left\{{ }^{1} \mathrm{H}\right\}$ NMR $\left(\mathrm{CDCl}_{3}\right): \delta 44.2\left(\mathrm{~s}, J_{\mathrm{Pt}-\mathrm{P}}=2754 \mathrm{~Hz}\right),-14.8(\mathrm{~s})$. UVvis $\left[\mathrm{CH}_{2} \mathrm{Cl}_{2} ; \lambda_{\max }, \mathrm{nm}\left(\varepsilon, \mathrm{M}^{-1} \mathrm{~cm}^{-1}\right)\right]: 418$ (3910). MS (ESI $\left.{ }^{+}\right)$. Calcd for monoisotopic $\left[\mathrm{C}_{58} \mathrm{H}_{48} \mathrm{P}_{4} \mathrm{PtS}_{2}\right]^{+}: \mathrm{m} / z$ 1128.1811. Obsd: $\mathrm{m} / \mathrm{z}$ 1128.1731. Error $(\delta): 7.11 \mathrm{ppm}$.

$\left[\left(\mathrm{Ph}_{2} \mathrm{C}_{2} \mathrm{~S}_{2}\right) \mathrm{Ni}(\mathrm{tpbz})\right](8)$. A $50 \mathrm{~mL}$ Schlenk flask with a stirbar was charged with tpbz $(0.081 \mathrm{~g}, 0.099 \mathrm{mmol})$ and $20 \mathrm{~mL}$ of $\mathrm{CH}_{2} \mathrm{Cl}_{2}$. Under an outward flow of $\mathrm{N}_{2},\left[\mathrm{Ni}\left(\mathrm{S}_{2} \mathrm{C}_{2} \mathrm{Ph}_{2}\right)_{2}\right](0.059 \mathrm{~g}, 0.11 \mathrm{mmol})$ was added, which quickly induced the formation of a dark-green color. The resulting mixture was stirred for $4 \mathrm{~h}$, after which time the solvent was removed under reduced pressure. The solid residual was purified on a silica column eluted with $2: 1 \mathrm{CH}_{2} \mathrm{Cl}_{2} /$ hexanes, and 8 was isolated as a green band. Following removal of the solvent under reduced pressure, recrystallization of $\mathbf{8}$ was accomplished by the diffusion of $\mathrm{Et}_{2} \mathrm{O}$ vapor into a filtered $\mathrm{CH}_{2} \mathrm{Cl}_{2}$ or $\mathrm{ClCH}_{2} \mathrm{CH}_{2} \mathrm{Cl}$ solution. Continued elution of the column with 10:1 $\mathrm{CH}_{2} \mathrm{Cl}_{2} / \mathrm{THF}$ led to a brown band of $[(\mathrm{pdt}) \mathrm{Ni}(\mathrm{tpbz}) \mathrm{Ni}(\mathrm{pdt})]$, the properties of which were reported earlier. ${ }^{2}$ Yield: $0.059 \mathrm{~g}, 0.053 \mathrm{mmol}, 53 \% . R_{f}=$ 0.82 (9:1 $\mathrm{CH}_{2} \mathrm{Cl}_{2} /$ hexanes). ${ }^{1} \mathrm{H}$ NMR $\left(\mathrm{CDCl}_{3}\right): \delta 7.48-7.42(\mathrm{~m}, 8 \mathrm{H}$, aromatic $\mathrm{C}-\mathrm{H}), 7.38-7.33(\mathrm{~m}, 4 \mathrm{H}$, aromatic $\mathrm{C}-\mathrm{H}), 7.24-7.20(\mathrm{~m}$, $16 \mathrm{H}$, aromatic $\mathrm{C}-\mathrm{H}), 7.13-7.08(\mathrm{~m}, 10 \mathrm{H}$, aromatic $\mathrm{C}-\mathrm{H}), 7.04-$ $7.00(\mathrm{~m}, 5 \mathrm{H}$, aromatic $\mathrm{C}-\mathrm{H}), 6.97-6.92(\mathrm{~m}, 9 \mathrm{H}$, aromatic $\mathrm{C}-\mathrm{H})$. ${ }^{31} \mathrm{P}\left\{{ }^{1} \mathrm{H}\right\}$ NMR $\left(\mathrm{CDCl}_{3}\right): \delta 55.0(\mathrm{~s}),-14.8(\mathrm{~s}) . \mathrm{UV}$-vis $\left[\mathrm{CH}_{2} \mathrm{Cl}_{2}\right.$; $\left.\lambda_{\max } \mathrm{nm}\left(\varepsilon, \mathrm{M}^{-1} \mathrm{~cm}^{-1}\right)\right]: 416$ (3680), 616 (560). MS (ESI $\left.{ }^{+}\right)$. Calcd for monoisotopic $\left[\mathrm{C}_{68} \mathrm{H}_{52} \mathrm{NiP}_{4} \mathrm{~S}_{2}\right]^{+}: \mathrm{m} / z$ 1114.1814. Obsd: $\mathrm{m} / \mathrm{z}$ 1114.178. Error $(\delta): 3.08 \mathrm{ppm}$. Anal. Calcd for $8\left(\mathrm{C}_{68} \mathrm{H}_{52} \mathrm{NiP}_{4} \mathrm{~S}_{2}\right): \mathrm{C}$, 73.19; H, 4.70; P, 11.10. Found: C, 72.99; H, 4.78; P, 11.24 .

$\left[\left(\mathrm{Ph}_{2} \mathrm{C}_{2} \mathrm{~S}_{2}\right) \mathrm{Pd}(\mathrm{tpbz})\right]$ (9). The same procedure and scale as those described for $\mathbf{8}$ were implemented, with the only differences being the onset of a reddish-brown color during the reaction and elution (with 2:1 $\mathrm{CH}_{2} \mathrm{Cl}_{2} /$ hexanes) of the product as a dark-red-purple band from the silica column. Recrystallization was accomplished by the diffusion of $\mathrm{Et}_{2} \mathrm{O}$ into a filtered $\mathrm{CH}_{2} \mathrm{Cl}_{2}$ solution. Continued elution of the column with 10:1 $\mathrm{CH}_{2} \mathrm{Cl}_{2}$.THF rapidly led to a dark-reddish-orange band of $[(\mathrm{pdt}) \mathrm{Pd}(\mathrm{tpbz}) \mathrm{Pd}(\mathrm{pdt})]$, which has been previously described. ${ }^{2}$ Yield: $0.060 \mathrm{~g}, 0.052 \mathrm{mmol}, 52 \% . \quad R_{f}=0.73$ (9:1 $\mathrm{CH}_{2} \mathrm{Cl}_{2}$ /hexanes). ${ }^{1} \mathrm{H}$ NMR $\left(\mathrm{CDCl}_{3}\right): \delta 7.45-7.34(\mathrm{~m}, 11 \mathrm{H}$, aromatic $\mathrm{C}-\mathrm{H}), 7.26-7.22(\mathrm{~m}, 20 \mathrm{H}$, aromatic $\mathrm{C}-\mathrm{H}), 7.13-7.08$ $(\mathrm{m}, 8 \mathrm{H}$, aromatic $\mathrm{C}-\mathrm{H}), 7.04-6.92(\mathrm{~m}, 13 \mathrm{H}$, aromatic $\mathrm{C}-\mathrm{H})$. ${ }^{31} \mathrm{P}\left\{{ }^{1} \mathrm{H}\right\} \operatorname{NMR}\left(\mathrm{CDCl}_{3}\right): \delta 49.4(\mathrm{~s}),-14.7(\mathrm{~s}) . \mathrm{UV}-$ vis $\left[\mathrm{CH}_{2} \mathrm{Cl}_{2}\right.$; $\left.\lambda_{\max } \mathrm{nm}\left(\varepsilon, \mathrm{M}^{-1} \mathrm{~cm}^{-1}\right)\right]: 420$ (2670), 544 (860). MS (ESI $\left.{ }^{+}\right)$. Calcd for monoisotopic $\left[\mathrm{C}_{68} \mathrm{H}_{52} \mathrm{P}_{4} \mathrm{PdS}_{2}\right]^{+}: \mathrm{m} / z$ 1162.1517. Obsd: $\mathrm{m} / z$ 1162.1485. Error $(\delta): 2.77 \mathrm{ppm}$. Anal. Calcd for $9\left(\mathrm{C}_{68} \mathrm{H}_{52} \mathrm{PdP}_{4} \mathrm{~S}_{2}\right)$ : C, 70.19; H, 4.50; P, 10.65. Found: C, 69.57; H, 4.17; P, 9.83.

[( $\left.\left.\mathrm{Ph}_{2} \mathrm{C}_{2} \mathrm{~S}_{2}\right) \mathrm{Pt}(\mathrm{tpbz})\right](11)$. Under an atmosphere of $\mathrm{N}_{2}$, a $50 \mathrm{~mL}$ Schlenk flask with a stirbar was charged with tpbz $(0.100 \mathrm{~g}, 0.123$ $\mathrm{mmol})$ and $\mathrm{CH}_{2} \mathrm{Cl}_{2}(20 \mathrm{~mL})$. Under an outward flow of $\mathrm{N}_{2}$, $\left[\mathrm{Pt}\left(\mathrm{S}_{2} \mathrm{C}_{2} \mathrm{Ph}_{2}\right)_{2}\right] \quad(0.0834 \mathrm{~g}, 0.123 \mathrm{mmol})$ was added, which immediately induced the formation of a blue color. Stirring was continued for $2 \mathrm{~h}$, and the reaction mixture was then kept for 3 days without stirring. The solvent was removed under reduced pressure, and the crude solid residue was purified on a silica column that was flash-eluted with 1:1 $\mathrm{CH}_{2} \mathrm{Cl}_{2} /$ hexanes. The title compound was collected as the leading yellow band. Crystallization of $\mathbf{1 1}$ as yellow columns was accomplished by the diffusion of $\mathrm{Et}_{2} \mathrm{O}$ vapor into a filtered $\mathrm{CH}_{2} \mathrm{Cl}_{2}$ solution. Yield: $0.057 \mathrm{~g}, 37 \% . R_{f}=0.77\left(9: 1 \mathrm{CH}_{2} \mathrm{Cl}_{2} /\right.$ hexanes). ${ }^{1} \mathrm{H}$ NMR $\left(\mathrm{CDCl}_{3}\right): \delta 7.60-7.51(\mathrm{~m}, 4 \mathrm{H}$, aromatic $\mathrm{C}-\mathrm{H})$, $7.39-7.36(\mathrm{~m}, 10 \mathrm{H}$, aromatic $\mathrm{C}-\mathrm{H}), 7.28-7.26(\mathrm{~m}, 8 \mathrm{H}$, aromatic $\mathrm{C}-\mathrm{H}), 7.06-7.01(\mathrm{~m}, 14 \mathrm{H}$, aromatic $\mathrm{C}-\mathrm{H}), 6.96-6.89(\mathrm{~m}, 16 \mathrm{H}$, aromatic $\mathrm{C}-\mathrm{H}) .{ }^{31} \mathrm{P}\left\{{ }^{1} \mathrm{H}\right\} \mathrm{NMR}\left(\mathrm{CDCl}_{3}\right): \delta 42.7\left(\mathrm{~s}, J_{\mathrm{Pt}-\mathrm{P}}=2742 \mathrm{~Hz}\right)$, -14.9 (s). UV-vis $\left[\mathrm{CH}_{2} \mathrm{Cl}_{2} ; \lambda_{\max } \mathrm{nm}\left(\varepsilon, \mathrm{M}^{-1} \mathrm{~cm}^{-1}\right)\right]: 332(8750)$, 399 (2750). MS $\left(\mathrm{ESI}^{+}\right)$. Calcd for monoisotopic $\left[\mathrm{C}_{68} \mathrm{H}_{52} \mathrm{P}_{4} \mathrm{PtS}_{2}\right]^{+}: \mathrm{m} /$ $z$ 1252.2126. Obsd: $m / z$ 1252.2067. Error $(\delta): 4.72 \mathrm{ppm}$. Anal. Calcd for $11\left(\mathrm{C}_{68} \mathrm{H}_{52} \mathrm{PtP}_{4} \mathrm{~S}_{2}\right)$ : C, 65.22; $\mathrm{H}, 4.19$. Found: C, 65.22; $\mathrm{H}, 4.13$.

$\left[\left(\mathrm{Ph}_{2} \mathrm{C}_{2} \mathrm{~S}_{2}\right)_{2} \mathrm{Pt}(\mathrm{tpbz})\right]$ (10). Continued elution of the column used for the isolation of compound 11 with $2: 1 \mathrm{CH}_{2} \mathrm{Cl}_{2} /$ hexanes moved 10 as a blue band, which was collected and reduced to dryness. Crystallization of $\mathbf{1 0}$ as blue blocks was achieved by the diffusion of $\mathrm{Et}_{2} \mathrm{O}$ vapor into a filtered $\mathrm{CH}_{2} \mathrm{Cl}_{2}$ solution. Yield: $0.017 \mathrm{~g}, 9 \% . \mathrm{R}_{f}=$ 0.23 (9:1 $\mathrm{CH}_{2} \mathrm{Cl}_{2} /$ hexanes), ${ }^{1} \mathrm{H}$ NMR $\left(\mathrm{CD}_{2} \mathrm{Cl}_{2}\right): \delta 7.64-7.48(\mathrm{~m}$, $8 \mathrm{H}$, aromatic $\mathrm{C}-\mathrm{H}), 7.40-7.19(\mathrm{~m}, 16 \mathrm{H}$, aromatic $\mathrm{C}-\mathrm{H}), 7.11-7.03$ $(\mathrm{m}, 12 \mathrm{H}$, aromatic $\mathrm{C}-\mathrm{H}), 6.96-6.93(\mathrm{~m}, 11 \mathrm{H}$, aromatic $\mathrm{C}-\mathrm{H})$, 6.92-6.84 (m, 9H, aromatic $\mathrm{C}-\mathrm{H}), 6.49-6.45(\mathrm{~m}, 16 \mathrm{H}$, aromatic 
$\mathrm{C}-H) .{ }^{31} \mathrm{P}\left\{{ }^{1} \mathrm{H}\right\}$ NMR $\left(\mathrm{CD}_{2} \mathrm{Cl}_{2}\right): \delta 19.0\left(\mathrm{~s}, J_{\mathrm{Pt}-\mathrm{P}}=1787 \mathrm{~Hz}\right),-13.3$ (s). UV-vis $\left[\mathrm{CH}_{2} \mathrm{Cl}_{2} ; \lambda_{\text {max }}, \mathrm{nm}\left(\varepsilon, \mathrm{M}^{-1} \mathrm{~cm}^{-1}\right)\right]: 583$ (2140), 355 (11500). MS (ESI ${ }^{+}$. Calcd for monoisotopic $\left[\mathrm{C}_{82} \mathrm{H}_{62} \mathrm{P}_{4} \mathrm{PtS}_{4}\right]^{+}: \mathrm{m} / z$ 1494.2349. Obsd: $m / z$ 1494.229. Error $(\delta): 3.95$ ppm.

[((MeO-p- $\left.\left.\left.\mathrm{C}_{6} \mathrm{H}_{4}\right)_{2} \mathrm{C}_{2} \mathrm{~S}_{2}\right) \mathrm{Ni}(\mathrm{tpbz})\right]$ (12). The same procedure and scale as those described for the synthesis of $\mathbf{8}$ were implemented but with $\left[\left(\left(\mathrm{MeO}-p-\mathrm{C}_{6} \mathrm{H}_{4}\right)_{2} \mathrm{C}_{2} \mathrm{~S}_{2}\right)_{2} \mathrm{Ni}\right]$ used in place of $\left[\left(\mathrm{Ph}_{2} \mathrm{C}_{2} \mathrm{~S}_{2}\right)_{2} \mathrm{Ni}\right]$. Crystals were grown by the diffusion of hexanes vapor into a filtered chlorobenzene solution. Continued elution of the silica column used to purify 12 using 10:1 $\mathrm{CH}_{2} \mathrm{Cl}_{2}$ /THF rapidly moved a brown band of $\left[\left(\left(\mathrm{MeO}-p-\mathrm{C}_{6} \mathrm{H}_{4}\right)_{2} \mathrm{C}_{2} \mathrm{~S}_{2}\right) \mathrm{Ni}(\mathrm{tpbz}) \mathrm{Ni}\left(\mathrm{S}_{2} \mathrm{C}_{2}\left(\mathrm{C}_{6} \mathrm{H}_{4} \text { - } p \text {-OMe }\right)_{2}\right)\right]$, which has been previously described. ${ }^{2}$ Yield: $0.057 \mathrm{~g}$ of a dark-green solid, 49\%. $R_{f}=0.42$ (9:1 $\mathrm{CH}_{2} \mathrm{Cl}_{2} /$ hexanes). ${ }^{1} \mathrm{H}$ NMR $\left(\mathrm{CDCl}_{3}\right): \delta 7.50-$ $7.35(\mathrm{~m}, 14 \mathrm{H}$, aromatic $\mathrm{C}-\mathrm{H}), 7.26-7.19(\mathrm{~m}, 14 \mathrm{H}$, aromatic $\mathrm{C}-\mathrm{H})$, $7.16-7.10(\mathrm{~m}, 11 \mathrm{H}$, aromatic $\mathrm{C}-\mathrm{H}), 6.99-6.94(\mathrm{~m}, 7 \mathrm{H}$, aromatic $\mathrm{C}-\mathrm{H}), 6.63-6.60(\mathrm{~m}, 4 \mathrm{H}$, aromatic $\mathrm{C}-\mathrm{H}), 3.71(\mathrm{~s}, 6 \mathrm{H},-\mathrm{OMe})$. ${ }^{31} \mathrm{P}\left\{{ }^{1} \mathrm{H}\right\}$ NMR $\left(\mathrm{CDCl}_{3}\right): \delta 55.2(\mathrm{~s}),-14.2(\mathrm{~s}) . \mathrm{UV}-\mathrm{vis}\left[\mathrm{CH}_{2} \mathrm{Cl}_{2}\right.$; $\left.\lambda_{\max } \mathrm{nm}\left(\varepsilon, \mathrm{M}^{-1} \mathrm{~cm}^{-1}\right)\right]: 432$ (2860), 620 (610). MS (ESI $\left.{ }^{+}\right)$. Calcd for monoisotopic $\left[\mathrm{C}_{70} \mathrm{H}_{56} \mathrm{NiO}_{2} \mathrm{P}_{4} \mathrm{~S}_{2}\right]^{+}: \mathrm{m} / z$ 1174.2026. Obsd: $\mathrm{m} / z$ 1174.1936. Error $(\delta): 7.67 \mathrm{ppm}$. Anal. Calcd for 12 $\left(\left[\mathrm{C}_{70} \mathrm{H}_{56} \mathrm{NiO}_{2} \mathrm{P}_{4} \mathrm{~S}_{2}\right]\right): \mathrm{C}, 71.50 ; \mathrm{H}, 4.80 ; \mathrm{P}, 10.54 ; \mathrm{S}, 5.45$. Found: C, 71.29; H, 4.86; P, 10.41; S, 5.51.

[((MeO-p- $\left.\left.\left.\mathrm{C}_{6} \mathrm{H}_{4}\right)_{2} \mathrm{C}_{2} \mathrm{~S}_{2}\right) \mathrm{Pd}(\mathrm{tpbz})\right]$ (13). The same procedure and scale as those described for the synthesis of $\mathbf{8}$ were implemented but with $\left[\left(\left(\mathrm{MeO}-p-\mathrm{C}_{6} \mathrm{H}_{4}\right)_{2} \mathrm{C}_{2} \mathrm{~S}_{2}\right)_{2} \mathrm{Pd}\right]$ used in place of $\left[\left(\mathrm{Ph}_{2} \mathrm{C}_{2} \mathrm{~S}_{2}\right)_{2} \mathrm{Ni}\right]$. The crude solid residue was purified on a silica column that was flasheluted with 2:1 $\mathrm{CH}_{2} \mathrm{Cl}_{2} /$ hexanes; 13 was collected as the leading redpurple band. Continued elution with $10: 1 \mathrm{CH}_{2} \mathrm{Cl}_{2} / \mathrm{THF}$ rapidly led to a brown-purple band of $\left[\left(\left(\mathrm{MeO}-p-\mathrm{C}_{6} \mathrm{H}_{4}\right)_{2} \mathrm{C}_{2} \mathrm{~S}_{2}\right) \mathrm{Pd}(\mathrm{tpbz}) \mathrm{Pd}\right.$ $\left.\left(\mathrm{S}_{2} \mathrm{C}_{2}\left(\mathrm{C}_{6} \mathrm{H}_{4}-p-\mathrm{OMe}\right)_{2}\right)\right]$, which has been previously described. ${ }^{2}$ Yield: $0.063 \mathrm{~g}$ of a brown-red solid, $52 \% . R_{f}=0.23\left(9: 1 \mathrm{CH}_{2} \mathrm{Cl}_{2} /\right.$ hexanes). ${ }^{1} \mathrm{H}$ NMR $\left(\mathrm{CDCl}_{3}\right): \delta 7.47-7.35(\mathrm{~m}, 13 \mathrm{H}$, aromatic $\mathrm{C}-\mathrm{H})$, $7.28-7.21(\mathrm{~m}, 16 \mathrm{H}$, aromatic $\mathrm{C}-\mathrm{H}), 7.17-7.10(\mathrm{~m}, 10 \mathrm{H}$, aromatic $\mathrm{C}-\mathrm{H})$, 6.99-6.94 $(\mathrm{m}, 7 \mathrm{H}$, aromatic $\mathrm{C}-\mathrm{H}), 6.60-6.30(\mathrm{~m}, 4 \mathrm{H}$, aromatic $\mathrm{C}-\mathrm{H}), 3.70(\mathrm{~s}, 6 \mathrm{H},-\mathrm{OMe}) .{ }^{31} \mathrm{P}\left\{{ }^{1} \mathrm{H}\right\} \mathrm{NMR}\left(\mathrm{CDCl}_{3}\right): \delta 49.3$ (s), -14.7 (s). UV-vis $\left[\mathrm{CH}_{2} \mathrm{Cl}_{2} ; \lambda_{\max } \mathrm{nm}\left(\varepsilon, \mathrm{M}^{-1} \mathrm{~cm}^{-1}\right)\right]: 432$ (2180), 551 (930). MS $\left(\mathrm{ESI}^{+}\right)$. Calcd for monoisotopic $\left[\mathrm{C}_{70} \mathrm{H}_{56} \mathrm{O}_{2} \mathrm{P}_{4} \mathrm{PdS}_{2}\right]^{+}: \mathrm{m} / z$ 1221.1729. Obsd: $\mathrm{m} / z$ 1221.1652. Error $(\delta): 6.23 \mathrm{ppm}$.

[((MeO-p- $\left.\left.\left.\mathrm{C}_{6} \mathrm{H}_{4}\right)_{2} \mathrm{C}_{2} \mathrm{~S}_{2}\right) \mathrm{Pt}(\mathrm{tpbz})\right]$ (14). The same procedure and scale as those described for the synthesis of 8 were implemented but with $\left[\left(\left(\mathrm{MeO}-p-\mathrm{C}_{6} \mathrm{H}_{4}\right)_{2} \mathrm{C}_{2} \mathrm{~S}_{2}\right)_{2} \mathrm{Pd}\right]$ used in place of $\left[\left(\mathrm{Ph}_{2} \mathrm{C}_{2} \mathrm{~S}_{2}\right)_{2} \mathrm{Ni}\right]$. The crude solid residue was purified on a silica column eluted with 2:1 $\mathrm{CH}_{2} \mathrm{Cl}_{2} /$ hexanes, which led to the title compound as the leading yellow band. Continued elution with $10: 1 \mathrm{CH}_{2} \mathrm{Cl}_{2} / \mathrm{THF}$ rapidly led to $\mathrm{a}$ red band of $\left[\left(\left(\mathrm{MeO}-p-\mathrm{C}_{6} \mathrm{H}_{4}\right)_{2} \mathrm{C}_{2} \mathrm{~S}_{2}\right) \mathrm{Pt}(\mathrm{tpbz}) \mathrm{Pt}\left(\mathrm{S}_{2} \mathrm{C}_{2}\left(\mathrm{C}_{6} \mathrm{H}_{4}-p\right.\right.\right.$ $\left.\mathrm{OMe})_{2}\right)$ ]. Yield: $0.046 \mathrm{~g}$ of a bright-yellow solid, $35 \% . R_{f}=0.34$ (9:1 $\mathrm{CH}_{2} \mathrm{Cl}_{2} /$ hexanes). ${ }^{1} \mathrm{H}$ NMR $\left(\mathrm{CDCl}_{3}\right): \delta 7.41-7.35(\mathrm{~m}, 8 \mathrm{H}$, aromatic $\mathrm{C}-\mathrm{H}), 7.29-7.25(\mathrm{~m}, 6 \mathrm{H}$, aromatic $\mathrm{C}-\mathrm{H}), 7.19-7.10(\mathrm{~m}$, $16 \mathrm{H}$, aromatic $\mathrm{C}-\mathrm{H}), 7.09-7.01(\mathrm{~m}, 10 \mathrm{H}$, aromatic $\mathrm{C}-\mathrm{H}), 6.90-$ $6.85(\mathrm{~m}, 8 \mathrm{H}$, aromatic $\mathrm{C}-\mathrm{H}), 3.61(\mathrm{~s}, 6 \mathrm{H},-\mathrm{OMe}) .{ }^{31} \mathrm{P}\left\{{ }^{1} \mathrm{H}\right\}$ NMR $\left(\mathrm{CDCl}_{3}\right): \delta 43.8\left(\mathrm{~s}, J_{\mathrm{Pt}-\mathrm{P}}=2742 \mathrm{~Hz}\right),-14.8(\mathrm{~s}) . \mathrm{UV}-$ vis $\left[\mathrm{CH}_{2} \mathrm{Cl}_{2}\right.$; $\left.\lambda_{\max } \mathrm{nm}\left(\varepsilon, \mathrm{M}^{-1} \mathrm{~cm}^{-1}\right)\right]: 414$ (4140). MS $\left(\mathrm{ESI}^{+}\right)$. Calcd for monoisotopic $\left[\mathrm{C}_{70} \mathrm{H}_{56} \mathrm{O}_{2} \mathrm{P}_{4} \mathrm{PtS}_{2}\right]^{+}: \mathrm{m} / z$ 1312.2338. Obsd: $\mathrm{m} / z$ 1312.2294. Error $(\delta): 3.33 \mathrm{ppm}$.

$\left[\left(\mathrm{Ph}_{2} \mathrm{C}_{2} \mathrm{~S}_{2}\right) \mathrm{Pt}(\mathrm{tpbz}) \mathrm{Ni}\left(\mathrm{S}_{2} \mathrm{C}_{2} \mathrm{Me}_{2}\right)\right]$ (15). A $50 \mathrm{~mL}$ Schlenk flask was charged with $\left[\mathrm{Cl}_{2} \mathrm{Ni}(\mathrm{dme})\right](0.022 \mathrm{~g}, 0.1 \mathrm{mmol})$ and $11(0.125 \mathrm{~g}, 0.1$ mmol) under a $\mathrm{N}_{2}$ atmosphere. To this mixture of solids was added $\mathrm{CH}_{2} \mathrm{Cl}_{2}(20 \mathrm{~mL})$ via a syringe, and the resulting solution was stirred at room temperature for $1 \mathrm{~h}$. During this time, a red color appeared. To the same reaction mixture was added $\left[\left(\mathrm{Me}_{2} \mathrm{C}_{2} \mathrm{~S}_{2}\right) \mathrm{Sn}^{n} \mathrm{Bu}_{2}\right](0.035$ g, $0.1 \mathrm{mmol})$, and a deep-red color was generated. The reaction mixture was stirred at ambient temperature overnight $(12 \mathrm{~h})$, after which time the solvent was removed under reduced pressure. The resulting dark-red solid residue was triturated with stirring under $\mathrm{MeOH}(8 \mathrm{~mL})$. This $\mathrm{MeOH}$ washing was removed by filter cannulation, and the residue was washed again with $\mathrm{MeOH}$ (5 $\mathrm{mL})$, followed by $\mathrm{Et}_{2} \mathrm{O}(2 \times 5 \mathrm{~mL})$. Crude 15 was purified on a silica column eluted with $\mathrm{CH}_{2} \mathrm{Cl}_{2}$. The red band of 15 , following reduction to dryness, was crystallized from $\mathrm{ClCH}_{2} \mathrm{CH}_{2} \mathrm{Cl} /{ }^{t} \mathrm{BuOMe}$. Yield: 0.051 g of a red solid, $36 \% . R_{f}=0.18\left(\mathrm{CH}_{2} \mathrm{Cl}_{2}\right) .{ }^{1} \mathrm{H}$ NMR $\left(\mathrm{CDCl}_{3}\right): \delta 7.47-$ $7.41(\mathrm{~m}, 18 \mathrm{H}$, aromatic $\mathrm{C}-\mathrm{H}), 7.37-7.30(\mathrm{~m}, 9 \mathrm{H}$, aromatic $\mathrm{C}-\mathrm{H})$, 7.24-7.21 $(\mathrm{m}, 9 \mathrm{H}$, aromatic $\mathrm{C}-\mathrm{H}), 7.17-7.13(\mathrm{~m}, 11 \mathrm{H}$, aromatic $\mathrm{C}-\mathrm{H}), 6.99-6.96(\mathrm{~m}, 5 \mathrm{H}, 2 \mathrm{H}$, aromatic $\mathrm{C}-\mathrm{H}), 2.01\left(\mathrm{~s}, 6 \mathrm{H},-\mathrm{CH}_{3}\right)$. ${ }^{31} \mathrm{P}\left\{{ }^{1} \mathrm{H}\right\}$ NMR $\left(\mathrm{CDCl}_{3}\right): \delta 53.1(\mathrm{~s}), 42.3\left(\mathrm{~s}, J_{\mathrm{Pt}-\mathrm{P}}=2734 \mathrm{~Hz}\right)$. UVvis $\left[\mathrm{CH}_{2} \mathrm{Cl}_{2} ; \lambda_{\max } \mathrm{nm}\left(\varepsilon, \mathrm{M}^{-1} \mathrm{~cm}^{-1}\right)\right]: 466$ (3940), 733 (1030). MS $\left(\mathrm{ESI}^{+}\right)$. Calcd for monoisotopic $\left[\mathrm{C}_{72} \mathrm{H}_{58} \mathrm{NiP}_{4} \mathrm{PtS}_{4}\right]^{+}: \mathrm{m} / z$ 1428.1375. Obsd: $m / z$ 1428.1367. Error $(\delta): 0.56 \mathrm{ppm}$.

$\left[\left(\mathrm{Ph}_{2} \mathrm{C}_{2} \mathrm{~S}_{2}\right) \mathrm{Ni}\left(\mathrm{tpbzO}_{2}\right)\right]$ (16). In the open air, a solution of 8 $(0.111 \mathrm{~g}, 0.1 \mathrm{mmol})$ in $15 \mathrm{~mL}$ of $\mathrm{CHCl}_{3}$ was treated with a solution of $\mathrm{I}_{2}(0.051 \mathrm{~g}, 0.2 \mathrm{mmol})$ in $\mathrm{CHCl}_{3}(5 \mathrm{~mL})$. The reaction mixture was stirred overnight $(14 \mathrm{~h})$ at ambient temperature. The brownish solution was transferred to a separatory funnel and washed one time with aqueous $1 \mathrm{M} \mathrm{NaOH}(20 \mathrm{~mL})$. The organic phase was separated as a green solution from the aqueous phase and taken to dryness under reduced pressure to afford crude $\mathbf{1 6}$ as a dark-green solid. Further purification was performed by eluting a slurry of the crude mixture with 20:80 THF/ $\mathrm{CHCl}_{3}$ from a silica column; 16 led to a green band and was collected and recrystallized from $\mathrm{CH}_{2} \mathrm{Cl}_{2} / \mathrm{Et}_{2} \mathrm{O}$. Yield: $0.044 \mathrm{~g}$ of a green solid, $39 \% . R_{f}=0.18\left(8: 2 \mathrm{CH}_{2} \mathrm{Cl}_{2} / \mathrm{THF}\right) .{ }^{1} \mathrm{H}$ NMR $\left(\mathrm{CDCl}_{3}\right): \delta 7.42-7.36(\mathrm{~m}, 10 \mathrm{H}$, aromatic $\mathrm{C}-\mathrm{H}), 7.33-7.28$ $(\mathrm{m}, 10 \mathrm{H}$, aromatic $\mathrm{C}-\mathrm{H}), 7.26-7.21(\mathrm{~m}, 14 \mathrm{H}$, aromatic $\mathrm{C}-\mathrm{H})$, 7.16-7.12 $(\mathrm{m}, 13 \mathrm{H}$, aromatic $\mathrm{C}-\mathrm{H}), 6.98-6.94(\mathrm{~m}, 5 \mathrm{H}$, aromatic $\mathrm{C}-\mathrm{H}) .{ }^{31} \mathrm{P}\left\{{ }^{1} \mathrm{H}\right\}$ NMR $\left(\mathrm{CDCl}_{3}\right): \delta 55.8(\mathrm{~s}), 29.1(\mathrm{~s})$. UV-vis $\left[\mathrm{CH}_{2} \mathrm{Cl}_{2} ; \lambda_{\max }, \mathrm{nm}\left(\varepsilon, \mathrm{M}^{-1} \mathrm{~cm}^{-1}\right)\right]: 432$ (2340), 630 (660). IR $\left(\mathrm{CH}_{2} \mathrm{Cl}_{2}, \mathrm{~cm}^{-1}\right): 1245\left(\mathrm{vs}, \nu_{\mathrm{P}=\mathrm{O}}\right)$. MS $\left(\mathrm{ESI}^{+}\right)$. Calcd for monoisotopic $\left[\mathrm{C}_{68} \mathrm{H}_{52} \mathrm{NiO}_{2} \mathrm{P}_{4} \mathrm{~S}_{2}\right]^{+}: \mathrm{m} / z$ 1146.1713. Obsd: $\mathrm{m} / z$ 1146.1743. Error $(\delta): 2.62 \mathrm{ppm}$.

$\left.\left[\left(\mathrm{Me}_{2} \mathrm{C}_{2} \mathrm{~S}_{2}\right) \mathrm{Pt}(\mathbf{t p b z O})_{2}\right)\right]$ (17). In a $25 \mathrm{~mL}$ Schlenk tube, $\left[\left(\left(\mathrm{CH}_{3}\right)_{2} \mathrm{C}_{2} \mathrm{~S}_{2}\right) \mathrm{Pt}(\mathrm{tpbz})\right](0.0601 \mathrm{~g}, 0.0533 \mathrm{mmol})$ was dissolved in $\mathrm{CH}_{2} \mathrm{Cl}_{2}(3 \mathrm{~mL})$ under $\mathrm{N}_{2}$. To this solution was added $\mathrm{H}_{2} \mathrm{O}_{2}(0.02$ $\mathrm{mL}, 30 \%$ in $\mathrm{H}_{2} \mathrm{O}, 0.2 \mathrm{mmol}$ ), and the mixture was stirred at room temperature overnight. The solvent and all volatiles were removed under reduced pressure, and the solid residual was redissolved in a minimal volume of $\mathrm{CH}_{2} \mathrm{Cl}_{2}$. To this $\mathrm{CH}_{2} \mathrm{Cl}_{2}$ solution were added hexanes $(15 \mathrm{~mL})$ to induce precipitation of an orange solid, which was then isolated by filtration and washed with hexanes $(3 \times 5 \mathrm{~mL})$. Recrystallization of $\mathbf{1 7}$ as orange needles was accomplished by the diffusion of $\mathrm{Et}_{2} \mathrm{O}$ vapor into a $\mathrm{CH}_{2} \mathrm{Cl}_{2}$ solution. Yield: $0.0245 \mathrm{~g}, 40 \%$. ${ }^{1} \mathrm{H}$ NMR $\left(\mathrm{CDCl}_{3}\right): \delta 7.47-7.21(\mathrm{~m}, 42 \mathrm{H}$, aromatic $\mathrm{C}-\mathrm{H}), 2.15(\mathrm{~s}$, $\left.6 \mathrm{H}, \mathrm{CH}_{3}\right) \cdot{ }^{31} \mathrm{P}\left\{{ }^{1} \mathrm{H}\right\} \mathrm{NMR}\left(\mathrm{CDCl}_{3}\right): \delta 45.1\left(\mathrm{~s}, \mathrm{PPh}_{2} \mathrm{Pt}, J_{\mathrm{P}-\mathrm{Pt}}=2755\right.$ $\mathrm{Hz}), 29.0\left(\mathrm{~s}, \mathrm{PPh}_{2} \mathrm{O}\right)$. MS $\left(\mathrm{ESI}^{+}\right)$. Calcd for monoisotopic $\left[\mathrm{C}_{58} \mathrm{H}_{48} \mathrm{O}_{2} \mathrm{P}_{4} \mathrm{PtS}_{2}\right]^{1+}: \mathrm{m} / z$ 1160.1604. Obsd: $\mathrm{m} / z$ 1160.1709. Error $(\delta): 9.05 \mathrm{ppm}$.

$\left[\left(\mathrm{Me}_{2} \mathrm{C}_{2} \mathrm{~S}_{2}\right) \mathrm{Ni}\left(\mathrm{tpbzS}_{2}\right)\right]$ (18). A solution of $\left[\left(\left(\mathrm{CH}_{3}\right)_{2} \mathrm{C}_{2} \mathrm{~S}_{2}\right) \mathrm{Ni}\right.$ $(\mathrm{tpbz})](0.0477 \mathrm{~g}, 0.048 \mathrm{mmol})$ in THF $(3.6 \mathrm{~mL})$ was treated with $\left[\mathrm{NH}_{4}\right]_{2} \mathrm{~S}\left(0.60 \mathrm{~mL}, 20 \%\right.$ in $\left.\mathrm{H}_{2} \mathrm{O}\right)$ and then allowed to stir at $\sim 50{ }^{\circ} \mathrm{C}$ for 3 days. The mixture was reduced to dryness under reduced pressure, redissolved in a minimal volume of $\mathrm{CHCl}_{3}$, and eluted from a silica column packed as a slurry in $\mathrm{CHCl}_{3}$. The leading green-brown band was collected and reduced to dryness, yielding 18 as a brown solid. Yield: $0.0223 \mathrm{~g}, 38 \% .{ }^{1} \mathrm{H}$ NMR $\left(\mathrm{CDCl}_{3}\right): \delta 7.51-7.30(\mathrm{~m}, 26 \mathrm{H}$, aromatic $\mathrm{C}-\mathrm{H}), 7.30-7.16(\mathrm{~m}, 16 \mathrm{H}$, aromatic $\mathrm{C}-\mathrm{H}), 2.06(\mathrm{~s}, 6 \mathrm{H}$ $\left.-\mathrm{CH}_{3}\right) \cdot{ }^{31} \mathrm{P} \mathrm{NMR}\left(\mathrm{CDCl}_{3}\right): \delta 55.9\left(\mathrm{~s},-\mathrm{Ph}_{2} \mathrm{PNi}\right), 46.9\left(\mathrm{~s},-\mathrm{Ph}_{2} \mathrm{PS}\right)$. $\mathrm{UV}-$ vis $\left[\mathrm{CH}_{2} \mathrm{Cl}_{2} ; \lambda_{\max }, \mathrm{nm}\left(\varepsilon, \mathrm{M}^{-1} \mathrm{~cm}^{-1}\right)\right]: 448(2270)$. MS $\left(\mathrm{ESI}^{+}\right)$. Calcd for monoisotopic $\left[\mathrm{C}_{58} \mathrm{H}_{48} \mathrm{NiP}_{4} \mathrm{~S}_{4}\right]^{+}: \mathrm{m} / z$ 1054.0943. Obsd: $m / z$ 1054.0951. Error $(\delta)$ : $0.82 \mathrm{ppm}$. Anal. Calcd for $\mathbf{1 8}$ $\left(\left[\mathrm{C}_{58} \mathrm{H}_{48} \mathrm{NiP}_{4} \mathrm{~S}_{4}\right]\right): \mathrm{C}, 65.98 ; \mathrm{H}, 4.58 ; \mathrm{P}, 11.73$. Found: C, 65.46; $\mathrm{H}, 4.44 ; \mathrm{P}, 10.7$.

$\left[\mathrm{Ni}(\mathrm{dppbO})_{3}\right]\left[\mathrm{I}_{3}\right]_{2}\left([19]\left[\mathrm{I}_{3}\right]_{2}\right)$. Under an atmosphere of $\mathrm{N}_{2}$, a 50 $\mathrm{mL}$ Schlenk flask with a stirbar was charged with $\mathrm{Ni}\left(\mathrm{NO}_{3}\right)_{2}(0.013 \mathrm{~g}$, $0.039 \mathrm{mmol})$ and THF $(20 \mathrm{~mL})$. Under an outward flow of $\mathrm{N}_{2}$, solid $\mathrm{dppbO}_{2}(0.056 \mathrm{mg}, 0.117 \mathrm{mmol})$ was added in a single portion, which induced the development of a very light-green solution. The reaction mixture was stirred overnight $(14 \mathrm{~h})$ at ambient temperature. Cesium triiodide $(0.040 \mathrm{~g}, 0.078 \mathrm{mmol})$ was added under an outward flow of $\mathrm{N}_{2}$, followed by the addition of $\mathrm{MeOH}(2 \mathrm{~mL})$. The resulting redbrown solution was stirred for $5 \mathrm{~h}$ at ambient temperature. Under reduced pressure, the solution was concentrated to a volume of $\sim 3$ $\mathrm{mL}$, whereupon $\mathrm{Et}_{2} \mathrm{O}(10 \mathrm{~mL})$ was added to precipitate the crude 


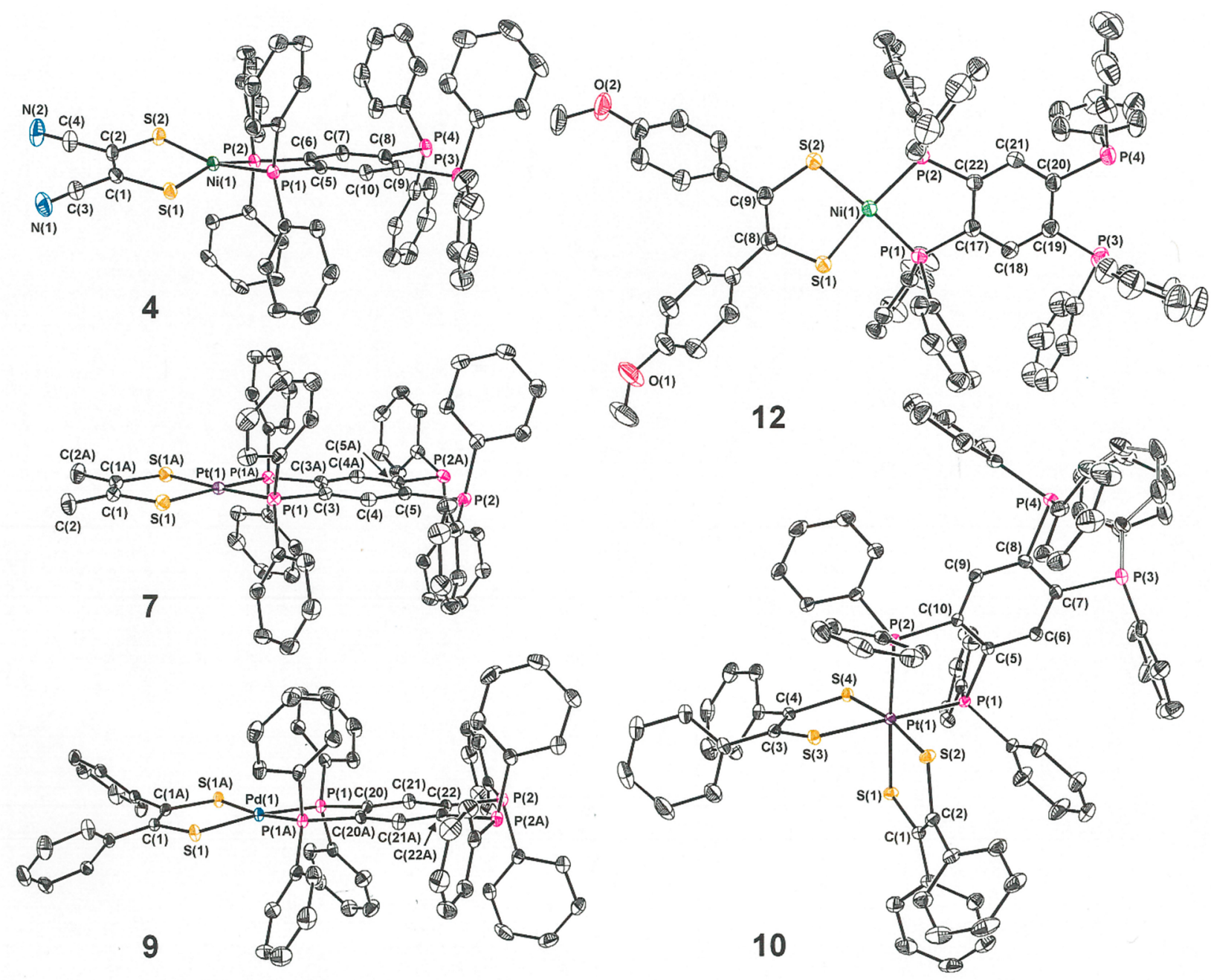

Figure 2. Thermal ellipsoid plots ( $50 \%$ probability level) of selected open-ended tpbz compounds that have been characterized by $\mathrm{X}$-ray crystallography. All $\mathrm{H}$ atoms have been omitted for clarity.

product as a red-brown powder. The solvent was removed by cannula filtration, and the dark red-brown solid residue was washed with $\mathrm{Et}_{2} \mathrm{O}$ $(3 \times 5 \mathrm{~mL})$ and dried under vacuum. Diffraction-quality red-orange plate crystals were grown by diffusion of $\mathrm{Et}_{2} \mathrm{O}$ into a filtered concentrate of the complex in $\mathrm{CH}_{2} \mathrm{Cl}_{2}$. Yield: $0.076 \mathrm{~g}, 87 \%$. UV-vis $\left[\mathrm{CH}_{2} \mathrm{Cl}_{2} ; \lambda_{\max } \mathrm{nm}\left(\varepsilon, \mathrm{M}^{-1} \mathrm{~cm}^{-1}\right)\right]: 426(8920)$. Solution IR $\left(\mathrm{CH}_{2} \mathrm{Cl}_{2}\right.$, $\left.\mathrm{cm}^{-1}\right): 1264$ (vs, $\left.\nu_{\mathrm{P}=\mathrm{O}}\right)$. MS $\left(\mathrm{ESI}^{+}\right)$. Calcd for monoisotopic $\left[\mathrm{C}_{90} \mathrm{H}_{72} \mathrm{NiP}_{6} \mathrm{~S}_{6}\right]^{+}: m / z$ 1492.3108. Calcd for $\left[\mathrm{C}_{90} \mathrm{H}_{72} \mathrm{NiP}_{6} \mathrm{~S}_{6}\right]^{2+}: m / z$ 746.1554. Obsd: $m / z$ 746.1566. Error $(\delta): 1.65 \mathrm{ppm}$.

\section{DISCUSSION}

Syntheses and Structures. Charge-neutral homoleptic bis(dithiolene) complexes of the Group 10 metals $\left[\mathrm{M}\left(\mathrm{S}_{2} \mathrm{C}_{2} \mathrm{R}_{2}\right)_{2}\right]\left(\mathrm{M}=\mathrm{Ni}^{2+}, \mathrm{Pd}^{2+}, \mathrm{Pt}^{2+}\right)$ are subject to direct displacement of one ligand by soft $\sigma$ donors such as phosphines or isocyanides. When the tpbz ligand is introduced to $\left[\mathrm{M}\left(\mathrm{S}_{2} \mathrm{C}_{2} \mathrm{R}_{2}\right)_{2}\right]$ in a $1: 1$ ratio, the open-ended $\left[\left[\left(\mathrm{R}_{2} \mathrm{C}_{2} \mathrm{~S}_{2}\right)\right.\right.$ $\mathrm{M}(\mathrm{tpbz})$ ] compounds are efficiently generated in yields ranging from $\sim 30$ to $50 \%$ (Scheme 2, Method 2). Purification by column chromatography and crystallization from dry solvents by vapor diffusion methods are straightforward. The Pt complexes proceed through an octahedral bis(dithiolene)diphosphine intermediate that is isolable in minor amounts (cf. 10, Scheme 2). Because the cyano-substituted dithiolene ligand does not support stable $\left[\mathrm{M}\left(\mathrm{S}_{2} \mathrm{C}_{2}(\mathrm{CN})_{2}\right)_{2}\right]^{0}$ complexes that can extrude a dithiolene ligand, the $\left[\left((\mathrm{NC}){ }_{2} \mathrm{C}_{2} \mathrm{~S}_{2}\right) \mathrm{M}\right.$ $(\mathrm{tpbz})]$ complexes must be prepared by the alternate route of transmetalation using a dialkyltin dithiolene complex (Scheme 2, Method 1). Method 1 has demonstrated usefulness in chloride-for-dithiolene ligand exchange for a broad variety of dithiolene ligand types. ${ }^{2,12-18}$ The open-ended $\left[\mathrm{Cl}_{2} \mathrm{M}(\mathrm{tpbz})\right]$ compounds $(1-3)$ with which these transmetalation reactions occur are themselves new compounds and are readily obtained in $\sim 80-90 \%$ yields by the introduction of tpbz to $\left[\mathrm{Cl}_{2} \mathrm{Ni}\right.$ (dme) $]$ or $\left[\mathrm{Cl}_{2} \mathrm{M}\left(\mathrm{N} \equiv \mathrm{CCH}_{3}\right)_{2}\right](\mathrm{M}=\mathrm{Pd}, \mathrm{Pt})$. All open-ended tpbz compounds are spectroscopically distinct by ${ }^{31} \mathrm{P}$ NMR from the symmetric, dimetallic compounds because they display a signal upfield of the $\mathrm{H}_{3} \mathrm{PO}_{4}$ reference, which is ascribed to the open end, and a signal $\sim 50 \mathrm{ppm}$ downfield of the reference, which arises from the $\mathrm{P}$ atoms chelated to $\mathrm{M}^{2+}$.

Representative members of the set of new compounds summarized in Scheme 2 have been characterized by X-ray crystallography (Figure 2 and Tables 1 and 2). In contrast to the dimetallic compounds $\left[\left(\mathrm{R}_{2} \mathrm{C}_{2} \mathrm{~S}_{2}\right) \mathrm{M}(\mathrm{tpbz}) \mathrm{M}\left(\mathrm{S}_{2} \mathrm{C}_{2} \mathrm{R}_{2}\right)\right]$, which show a proclivity to crystallize upon an inversion center in monoclinic $P 2_{1} / c$ (No. 14), the structures of the compounds in Scheme 2 reveal a tendency to coincide with $C_{2}$ axes in monoclinic $C 2 / c$ (No. 15). As is typical for the $\mathrm{d}^{8}$ configuration, compounds 1-5, 7-10, and 11 display structures that are best described as square-planar, albeit 
Table 2. Unit Cell and Refinement Data for Compounds Characterized by X-ray Diffraction

\begin{tabular}{|c|c|c|c|c|}
\hline compound & {$[(\mathrm{adt}) \mathrm{Ni}(\mathrm{tpbz})]$} & {$[(\mathrm{pdt}) \mathrm{Pt}(\mathrm{tpbz}) \mathrm{Ni}(\mathrm{mdt})]$} & {$\left[(\mathrm{pdt}) \mathrm{Ni}\left(\mathrm{tpbzO}_{2}\right)\right]$} & {$\left[(\mathrm{mdt}) \mathrm{Pt}\left(\mathrm{tpbzO}_{2}\right)\right]$} \\
\hline compound no. & 12 & 15 & 16 & 17 \\
\hline cocryst solvent & none & $2^{1} /{ }_{2}\left(\mathrm{ClCH}_{2} \mathrm{CH}_{2} \mathrm{Cl}\right)$ & $\mathrm{CH}_{2} \mathrm{Cl}_{2}$ & none \\
\hline formula & $\mathrm{C}_{70} \mathrm{H}_{56} \mathrm{NiO}_{2} \mathrm{P}_{4} \mathrm{~S}_{2}$ & $\mathrm{C}_{77} \mathrm{H}_{68} \mathrm{Cl}_{5} \mathrm{NiP}_{4} \mathrm{PtS}_{4}$ & $\mathrm{C}_{69} \mathrm{H}_{54} \mathrm{Cl}_{2} \mathrm{NiO}_{2} \mathrm{P}_{4} \mathrm{~S}_{2}$ & $\mathrm{C}_{58} \mathrm{H}_{48} \mathrm{O}_{2} \mathrm{P}_{4} \mathrm{PtS}_{2}$ \\
\hline $\mathrm{fw}, \mathrm{g} \mathrm{mol}^{-1}$ & 1175.85 & 1676.48 & 1232.73 & 1160.05 \\
\hline temperature, $\mathrm{K}$ & 150 & 150 & 150 & 150 \\
\hline wavelength, $\AA$ & 1.54178 & 0.71073 & 0.71073 & 0.71073 \\
\hline $2 \theta$ range, deg & $4.59-144.21$ & $3.87-66.84$ & $3.57-61.23$ & $4.18-66.45$ \\
\hline cryst syst & monoclinic & triclinic & triclinic & monoclinic \\
\hline space group & $P 2_{1} / n$ & $P \overline{1}$ & $P \overline{1}$ & $P 2_{1} / c$ \\
\hline a, $\AA$ & $12.0422(4)$ & $12.5374(6)$ & $13.4505(18)$ & $17.0751(11)$ \\
\hline$b, \AA$ & $38.5014(13)$ & $12.5692(6)$ & $16.597(2)$ & $16.0625(10)$ \\
\hline$c, \AA$ & $14.0003(6)$ & $24.7673(13)$ & $16.653(2)$ & $20.5241(13)$ \\
\hline$\alpha, \operatorname{deg}$ & 90 & $92.953(2)$ & $64.068(4)$ & 90 \\
\hline$\beta, \operatorname{deg}$ & $112.798(2)$ & $91.560(2)$ & $70.817(4)$ & $108.283(2)$ \\
\hline$\gamma, \operatorname{deg}$ & 90 & $108.166(2)$ & $72.092(4)$ & 90 \\
\hline volume $\left(\AA^{3}\right), Z$ & $5984.0(4), 4$ & 3699.6(3), 2 & 3098.4(7), 2 & $5345.0(6), 4$ \\
\hline density, $\mathrm{g} \mathrm{cm}^{-3}$ & 1.305 & 1.505 & 1.321 & 1.442 \\
\hline$\mu, \mathrm{mm}^{-1}$ & 2.484 & 2.565 & 0.615 & 2.864 \\
\hline color, habit & yellow-green plate & orange plate & orange plate & dark-orange column \\
\hline limiting indices $h$ & $-14 \leq h \leq 14$ & $-19<h<19$ & $-19<h<19$ & $-26 \leq h \leq 26$ \\
\hline limiting indices $k$ & $-44 \leq k \leq 46$ & $-19<k<19$ & $-23<k<23$ & $-24 \leq k \leq 24$ \\
\hline limiting indices $l$ & $-16 \leq l \leq 14$ & $0<l<38$ & $-23<1<23$ & $-31 \leq l \leq 31$ \\
\hline reflns collected & 51648 & 32993 & 144627 & 403235 \\
\hline indep data, param ${ }^{a}$ & 11399,703 & 32993,856 & 19027,728 & 20472,606 \\
\hline $\mathrm{GOF}^{b}$ & 1.039 & 1.277 & 1.044 & 1.081 \\
\hline $\mathrm{R} 1{ }^{c, d} \mathrm{wR}^{d, e}$ & $0.0479,0.1008$ & $0.0751,0.1691$ & $0.0488,0.1165$ & $0.0239,0.0594$ \\
\hline $\mathrm{R} 1,^{c, f} \mathrm{wR} 2^{e, f}$ & $0.0699,0.1106$ & $0.0827,0.1717$ & $0.0878,0.1350$ & $0.0314,0.0641$ \\
\hline compound & {$[(\mathrm{mdt}$} & & {$\left[\left(\mathrm{dppbO}_{2}\right)_{3} \mathrm{Ni}\right]\left[\mathrm{I}_{3}\right]_{2}$} & {$\left[\left(\mathrm{dppbO}_{2}\right)_{3} \mathrm{Ni}\right]\left[\mathrm{I}_{3}\right]_{2}$} \\
\hline compound no. & & & {$[19]\left[\mathrm{I}_{3}\right]_{2}$} & {$[19]\left[\mathrm{I}_{3}\right]_{2}$} \\
\hline cocryst solvent & & & none & none \\
\hline formula & & & $\mathrm{C}_{90} \mathrm{H}_{72} \mathrm{I}_{6} \mathrm{NiO}_{6} \mathrm{P}_{6}$ & $\mathrm{C}_{90} \mathrm{H}_{72} \mathrm{I}_{6} \mathrm{NiO}_{6} \mathrm{P}_{6}$ \\
\hline $\mathrm{fw}, \mathrm{g} \mathrm{mol}^{-1}$ & & & 2255.41 & 2255.40 \\
\hline temperature, $\mathrm{K}$ & & & 100 & 150 \\
\hline wavelength, $\AA$ & & & 0.71073 & 0.71073 \\
\hline $2 \theta$ range, deg & & & $3.12-54.00$ & $3.628-46.660$ \\
\hline cryst syst & & & triclinic & monoclinic \\
\hline space group & & & $P \overline{1}$ & $P 2_{1} / c$ \\
\hline a, $\AA$ & & & $13.0484(12)$ & $15.0552(10)$ \\
\hline$b, \AA$ & & & $13.3478(12)$ & $26.0258(18)$ \\
\hline$c, \AA$ & & & $26.880(3)$ & $22.6547(15)$ \\
\hline$\alpha, \operatorname{deg}$ & & & $102.137(1)$ & 90 \\
\hline$\beta, \operatorname{deg}$ & & & $92.504(1)$ & $101.217(2)$ \\
\hline$\gamma, \operatorname{deg}$ & & & $106.961(1)$ & 90 \\
\hline volume $\left(\AA^{3}\right), Z$ & & & $4350.1(7), 2$ & 8707.1(10), 4 \\
\hline density, $\mathrm{g} \mathrm{cm}^{-3}$ & & & 1.722 & 1.721 \\
\hline$\mu, \mathrm{mm}^{-1}$ & & & 2.514 & 2.512 \\
\hline color, habit & & & orange plate & orange-red plate \\
\hline limiting indices $h$ & -10 & & $-16 \leq h \leq 16$ & $-15 \leq h \leq 16$ \\
\hline limiting indices $k$ & $-3 s$ & & $-17 \leq k \leq 17$ & $-28 \leq k \leq 28$ \\
\hline limiting indices $l$ & -2 & & $-34 \leq l \leq 34$ & $-25 \leq l \leq 25$ \\
\hline reflns collected & & & 37066 & 153572 \\
\hline indep data, param ${ }^{a}$ & & & 18761,985 & 12505,989 \\
\hline $\mathrm{GOF}^{b}$ & & & 0.945 & 1.023 \\
\hline $\mathrm{R} 11^{c, d} \mathrm{wR}^{d, e}$ & 0.08 & & $0.0427,0.0931$ & $0.0505,0.1241$ \\
\hline $\mathrm{R} 11^{c_{f} f} \mathrm{wR}^{e_{f} f}$ & 0.17 & & $0.0707,0.1017$ & $0.0794,0.1403$ \\
\hline
\end{tabular}

${ }^{a}$ Independent data collected and parameters refined. ${ }^{b} \mathrm{GOF}=\left\{\sum\left[w\left(F_{\mathrm{o}}{ }^{2}-F_{\mathrm{c}}{ }^{2}\right)^{2}\right] /(n-p)\right\}^{1 / 2}$, where $n=$ number of reflections and $p$ is the total number of parameters refined. ${ }^{c} \mathrm{R} 1=\sum\left\|F_{\mathrm{o}}|-| F_{\mathrm{c}}\right\| / \sum\left|F_{\mathrm{o}}\right|{ }^{d} R$ indices for data cut off at $I>2 \sigma(I) .{ }^{e} \mathrm{wR} 2=\left\{\sum\left[w\left(F_{\mathrm{o}}{ }^{2}-F_{\mathrm{c}}{ }^{2}\right)^{2}\right] / \sum\left[w\left(\mathrm{~F}_{\mathrm{o}}{ }^{2}\right)^{2}\right]\right\}^{1 / 2} ; w=$ $1 /\left[\sigma^{2}\left(F_{\mathrm{o}}{ }^{2}\right)+(x P)^{2}+y P\right]$, where $P=\left[2 F_{\mathrm{c}}{ }^{2}+\operatorname{Max}\left(F_{\mathrm{o}}{ }^{2}, 0\right)\right] / 3 .{ }^{f_{R}}$ indices for all data.

with minor tetrahedral distortions. Quantified by the angle $\theta$ between the $\mathrm{S}-\mathrm{M}-\mathrm{S}$ and $\mathrm{P}-\mathrm{M}-\mathrm{P}$ planes, these departures from ideal planarity are more pronounced for the $\mathrm{Ni}$ complexes than for the heavier metal complexes (Table 3). 
Table 3. Selected Interatomic Distances, Bond Angles, And Other Structural Parameters for Compounds 1, 4, 5, 7, $\left[(\mathrm{pdt}) \mathrm{M}\left(\eta^{2}\right.\right.$-tpbz $\left.)\right](\mathrm{M}=\mathrm{Ni}, 8 ; \mathrm{Pd}, 9 ; \mathrm{Pt}, 11)$, and $12^{a}$

\begin{tabular}{|c|c|c|c|c|c|c|c|c|}
\hline & 1 & 4 & 5 & 7 & 8 & 9 & 11 & 12 \\
\hline$M-X^{b}$ & $2.1814[8]$ & $2.1489[6]$ & $2.1494(6)$ & $2.2933(8)$ & $2.1518(5)$ & $2.2935(6)$ & $2.2993(8)$ & $2.1457[6]$ \\
\hline $\mathrm{M}-\mathrm{P}^{c}$ & $2.1320[8]$ & $2.1542[6]$ & $2.1620(6)$ & $2.2431(8)$ & $2.1712(8)$ & $2.2713(2)$ & $2.2521(9)$ & $2.1503[6]$ \\
\hline$\Delta,{ }^{d} \AA$ & & 0.0053 & 0.0126 & -0.0502 & 0.0194 & -0.0222 & -0.0472 & 0.0046 \\
\hline$S-C$ & & $1.738[2]$ & $1.765(2)$ & $1.760(3)$ & $1.7568(16)$ & $1.767(2)$ & $1.767(3)$ & $1.757[2]$ \\
\hline $\mathrm{s}_{-} \mathrm{C}=\mathrm{C}_{-\mathrm{s}}$ & & $1.353(5)$ & $1.333(5)$ & $1.327(6)$ & $1.346(3)$ & $1.340(5)$ & $1.348(7)$ & $1.353(4)$ \\
\hline $\mathrm{P}-\mathrm{C}_{\text {bound }}{ }^{e}$ & $1.814[3]$ & $1.813[2]$ & $1.827(2)$ & $1.822(3)$ & $1.8242(16)$ & $1.825(2)$ & $1.820(3)$ & $1.827[2]$ \\
\hline $\mathrm{P}-\mathrm{C}_{\text {open }}{ }^{f}$ & $1.852[3]$ & $1.848[2]$ & $1.848(2)$ & $1.849(3)$ & $1.8449(16)$ & $1.849(2)$ & $1.840(3)$ & $1.843[2]$ \\
\hline $\mathrm{md},^{g} \AA$ & 0.144 & 0.267 & 0.070 & 0.035 & 0.125 & 0.112 & 0.112 & 0.243 \\
\hline$\delta,^{h} \AA$ & 0.019 & 0.028 & 0.000 & 0.000 & 0.000 & 0.000 & 0.000 & 0.056 \\
\hline $\mathrm{X}-\mathrm{M}-\mathrm{X}^{b}$ & $94.73(5)$ & $93.62(3)$ & $90.83(3)$ & $88.16(4)$ & $90.89(2)$ & $88.35(3)$ & $88.04(4)$ & $91.71(3)$ \\
\hline $\mathrm{P}-\mathrm{M}-\mathrm{P}^{c}$ & $88.68(2)$ & $87.65(3)$ & $89.38(3)$ & $87.74(4)$ & $88.64(4)$ & $86.50(3)$ & $86.80(4)$ & $89.57(3)$ \\
\hline $\mathrm{X}-\mathrm{M}-\mathrm{P}_{\mathrm{cis}}^{b}$ & $89.04[4]$ & $92.00[2]$ & $90.08(2)$ & $92.09(3)$ & $90.827(14)$ & $93.01(2)$ & $93.01(3)$ & $89.68[2]$ \\
\hline $\mathrm{X}-\mathrm{M}-\mathrm{P}_{\text {trans }}{ }^{b}$ & $169.96[4]$ & $162.19[3]$ & $175.30(2)$ & $177.75(3)$ & $171.658(16)$ & $172.88(8)$ & $172.92(3)$ & $163.90[3]$ \\
\hline$\theta,^{i} \operatorname{deg}$ & 13.1 & 24.9 & 6.6 & 3.2 & 11.7 & 10.2 & 10.2 & 22.5 \\
\hline$\varphi,{ }^{j} \operatorname{deg}$ & 3.5 & 7.2 & 1.2 & 1.2 & 2.6 & 2.4 & 2.5 & 1.9 \\
\hline
\end{tabular}

${ }^{a}$ Averaged values are given where two or more chemically identical interatomic distances or angles are present. Uncertainties are propagated according to Taylor, J. R. An Introduction to Error Analysis, 2nd ed.; University Science Books: Sausalito, CA, 1997; pp 73-77; propagated uncertainties are designated with [ ]. ${ }^{b} \mathrm{M}=\mathrm{Ni}, \mathrm{X}=\mathrm{Cl} ; \mathrm{M}=\mathrm{Ni}$, Pd, or Pt and $\mathrm{X}=\mathrm{S} .{ }^{c} \mathrm{M}=\mathrm{Ni}$, Pd, or Pt. ${ }^{d} \Delta=\mathrm{M}-\mathrm{P}-\mathrm{M}-\mathrm{S}$ bond length difference. ${ }^{e} \mathrm{C}$ atom of the central arene ring, metalated side. ${ }^{f} \mathrm{C}$ atom of the central arene ring, open side. ${ }^{g} \mathrm{md}=$ mean atom deviation from the $\mathrm{X}_{2} \mathrm{MP}{ }_{2}$ plane. ${ }^{h} \delta=$ deviation $(\AA)$ of $\mathrm{M}$ from the $\mathrm{X}_{2} \mathrm{MP}_{2}$ plane. ${ }^{i}$ Angle between the $\mathrm{S}_{2} \mathrm{M}$ and $\mathrm{P}_{2} \mathrm{M}$ planes. ${ }^{j}$ Angle between the $\mathrm{MP}_{2}$ and $\mathrm{P}_{2} \mathrm{C}_{6} \mathrm{P}_{2}$ mean planes.

Table 4. Selected Bond Lengths (̊) and Angles (deg) for $10^{a}$

$\begin{array}{ll}\mathrm{C}=\mathrm{C}_{\text {dithiolene,chelate }} & 1.348[3] \\ \mathrm{C}-\mathrm{S} & 1.771[2] \\ \mathrm{Pt}-\mathrm{S}_{\text {cis to }} \mathrm{P} & 2.3688[5] \\ \mathrm{Pt}-\mathrm{S}_{\text {trans to }} & 2.3607[5] \\ \mathrm{Pt}-\mathrm{P} & 2.3373[5] \\ \mathrm{S}(2)-\mathrm{Pt}(1)-\mathrm{S}(4) & 170.34(2) \\ \mathrm{S}-\mathrm{Pt}-\mathrm{S}_{\text {intraligand }} & 88.63[1] \\ \mathrm{S}(1)-\mathrm{Pt}(1)-\mathrm{S}(3) & 91.23(3) \\ \mathrm{S}-\mathrm{Pt}-\mathrm{S}^{b} & 84.63[1] \\ \mathrm{P}-\mathrm{Pt}-\mathrm{P} & 86.09(2) \\ \varphi^{c} & 15.9\end{array}$

${ }^{a}$ Averaged values are given where two or more chemically identical interatomic distances or angles are present. Uncertainties are propagated according to Taylor, J. R. An Introduction to Error Analysis, 2nd ed.; University Science Books: Sausalito, CA, 1997; pp 73-77; propagated uncertainties are designated with []$.{ }^{b}$ Interligand $\mathrm{S}-\mathrm{Pt}-\mathrm{S}$, with one $\mathrm{S}$ atom in the $\mathrm{PtP}_{2}$ plane and one orthogonal to it. ${ }^{c} \varphi=$ angle between the $\mathrm{PtP}_{2}$ plane and $\mathrm{P}_{2} \mathrm{C}_{6} \mathrm{P}_{2}$ mean plane.

A longer $\mathrm{M}-\mathrm{P}$ then $\mathrm{M}-\mathrm{S}$ bond length is observed in all $\mathrm{Ni}$ compounds, while the inverse is true is for the $\mathrm{Pd}$ and $\mathrm{Pt}$ complexes. This same pattern is clearly evident in the $\left[\left(\mathrm{R}_{2} \mathrm{C}_{2} \mathrm{~S}_{2}\right) \mathrm{M}(\mathrm{tpbz}) \mathrm{M}\left(\mathrm{S}_{2} \mathrm{C}_{2} \mathrm{R}_{2}\right)\right]$ compounds and has been attributed to more effective metal $\mathrm{d}-$ sulfur $\mathrm{p} \sigma$ overlap in the HOMO-3 and HOMO-4 for $\mathrm{Ni}$ versus $\mathrm{Pd}$ and $\mathrm{Pt}^{2}$ Undoubtedly, this explanation is equally pertinent to the openended compounds. The $\mathrm{P}-\mathrm{C}$ bond lengths to the central arene ring of the tpbz ligand differ notably between the bound and open ends of the ligand. A consistently longer $\mathrm{P}-\mathrm{C}$ bond, by $\sim 0.02 \AA$, is found at the open end.

Bis(dithiolene)diphosphine compound $\mathbf{1 0}$ is an intermediate on the pathway toward $\mathbf{1 1}$ and is isolable in varying amounts depending the temperature and length of the reaction time. Compound 10 occurs as a result of an atypical oxidative addition reaction, wherein the two electrons from $\mathrm{Pt}^{\mathrm{II}}$ in $\left[\left(\mathrm{Ph}_{2} \mathrm{C}_{2} \mathrm{~S}_{2}\right)_{2} \mathrm{Pt}\right]$ are distributed to the dithiolene radical monoanions $(\mathbf{b} \rightarrow \mathbf{a}$, Scheme 1$)$, thereby reducing them to ene-1,2-dithiolates rather than to the incoming ligand (diphosphine), as is usually the case. The subsequent transformation of $\mathbf{1 0}$ to $\mathbf{1 1}$ is a reductive elimination that extrudes one dithiolene ligand in a highly reactive fully oxidized form (c or possibly $\mathbf{d}$, Scheme 1 ) and restores the $\mathrm{Pt}^{\mathrm{II}}$ redox state. In related systems, trapped forms of this expelled dithiolene ligand, e.g., as a 1,2,5,6-tetrathiocin ${ }^{19}$ or as a 1,3dithiol-2-alkylimine, ${ }^{20}$ affirm this general accounting of the redox transaction. The crystal structure of $\mathbf{1 0}$ (Figure 2) establishes the octahedral geometry that is common for $\mathrm{Pt}^{\mathrm{IV}}$, with both dithiolene ligands in the fully reduced ene-1,2dithiolate redox level, as gauged by $\mathrm{S}-\mathrm{C}$ and $\mathrm{C}-\mathrm{C}_{\text {chelate }}$ bond lengths (Table 4). These $\mathrm{S}-\mathrm{C}$ and $\mathrm{C}-\mathrm{C}_{\text {chelate }}$ bond lengths are markedly longer and shorter, respectively, than those in the initial $\left[\left(\mathrm{Ph}_{2} \mathrm{C}_{2} \mathrm{~S}_{2}\right)_{2} \mathrm{Pt}\right]$ complex. Furthermore, UV-vis and $\mathrm{X}$ ray absorption spectroscopic measurements of very similar platinum dithiolenebis(phosphine) complexes ${ }^{21}$ are consistent with the foregoing descriptions of the redox changes and molecular electronic structure.

Heterodimetallic compound $\mathbf{1 5}$ is readily prepared either by the treatment of 11 with $\left[\left(\mathrm{Me}_{2} \mathrm{C}_{2} \mathrm{~S}_{2}\right)_{2} \mathrm{Ni}\right]$ or by the reaction between 5 and $\left[\left(\mathrm{Ph}_{2} \mathrm{C}_{2} \mathrm{~S}_{2}\right)_{2} \mathrm{Pt}\right]$ (Scheme 3$)$. This flexibility in the approach to the synthesis is enabled by the robust character of the open-ended tpbz compounds and offers a glimmer of future possibilities for "modular" synthesis. The structure of $\mathbf{1 5}$ (Figure 3 and Table 5) reveals bond lengths and other parameters that are consistent with independent, noninteracting metal centers. For example, the $\Delta_{M}$ values (the difference between the $\mathrm{M}-\mathrm{P}$ and $\mathrm{M}-\mathrm{S}$ average bond lengths) are negative and positive, respectively, for $\mathrm{Pt}$ and $\mathrm{Ni}$, as found for the analogous homodimetallic compounds. ${ }^{2}$ The Ni center reveals a slight tetrahedralization $\left(\varphi=11.4^{\circ}\right)$ compared to the more planar environment around $\operatorname{Pt}\left(\varphi=3.4^{\circ}\right)$.

As suggested by the synthesis of 15 , the open-ended compounds of Scheme 2 are themselves phosphine ligands that should broadly manifest the reactivity associated with this ligand type. As one example, without detriment to the 


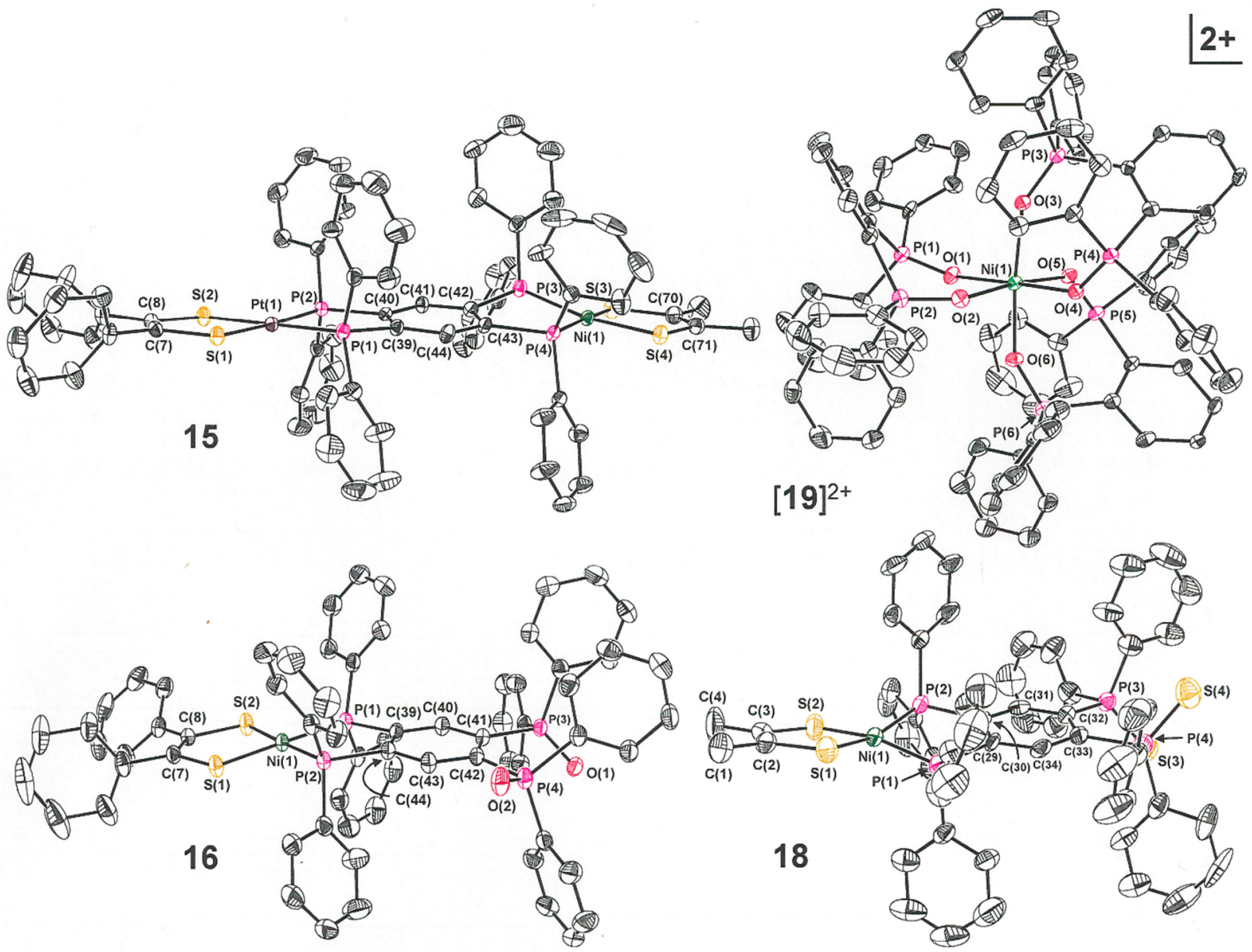

Figure 3. Thermal ellipsoid plots (50\% probability level) of dimetallic 15, open-ended oxidized 16 and $\mathbf{1 8}$, and mononuclear $[\mathbf{1 9}]^{2+}$ from X-ray crystallographic characterization. All $\mathrm{H}$ atoms have been omitted for clarity.

Table 5. Selected Interatomic Distances $(\AA)$ and Angles (deg) for $15^{a}$

$\begin{array}{llll}\mathrm{Pt}-\mathrm{S} & 2.2921[12] & \mathrm{S}-\mathrm{Pt}-\mathrm{S} & 88.81(6) \\ \mathrm{Pt}-\mathrm{P} & 2.2461[12] & \mathrm{P}-\mathrm{Pt}-\mathrm{P} & 87.43(6) \\ \Delta_{\mathrm{Pt}}{ }^{b} & -0.046 & \mathrm{~S}-\mathrm{Pt}-\mathrm{P}_{\text {cis }} & 91.88[4] \\ \mathrm{Ni}-\mathrm{S} & 2.142[1] & \mathrm{S}-\mathrm{Pt}-\mathrm{P}_{\text {trans }} & 177.68[5] \\ \mathrm{Ni}-\mathrm{P} & 2.1535[13] & \theta_{1}{ }^{c} \text { deg } & 3.4 \\ \Delta_{\mathrm{Ni}}{ }^{b} & +0.012 & \mathrm{~S}-\mathrm{Ni}-\mathrm{S} & 91.21(8) \\ \mathrm{S}-\mathrm{C}_{\mathrm{pdt}} & 1.758[4] & \mathrm{P}-\mathrm{Ni}-\mathrm{P} & 88.07(7) \\ \mathrm{S}-\mathrm{C}_{\text {mdt }} & 1.751[5] & \mathrm{S}-\mathrm{Ni}-\mathrm{P}_{\text {cis }} & 90.82[5] \\ \mathrm{C}=\mathrm{C}_{\mathrm{pdt}} & 1.350(9) & \mathrm{S}-\mathrm{Ni}-\mathrm{P}_{\text {trans }} & 171.68[6] \\ \mathrm{C}=\mathrm{C}_{\text {mdt }} & 1.352(11) & \theta_{2}{ }^{c}{ }^{\mathrm{deg}} & 11.4\end{array}$

Averaged values are given where two or more chemically identical interatomic distances or angles are present. Uncertainties are propagated according to Taylor, J. R. An Introduction to Error Analysis, 2nd ed.; University Science Books: Sausalito, CA, 1997; pp 73-77; propagated uncertainties are designated with [ ]. ${ }^{b} \Delta_{\mathrm{M}}=$ $(\mathrm{M}-\mathrm{P})-(\mathrm{M}-\mathrm{S})$ bond length difference. ${ }^{c} \theta=$ angle between the $\mathrm{S}_{2} \mathrm{M}$ and $\mathrm{P}_{2} \mathrm{M}$ planes.

metalated end of tpbz, the open phosphine groups can undergo oxidative addition of the chalcogen atom to form the corresponding phosphine oxides or sulfides. Several representative compounds $-\left[\left(\mathrm{Ph}_{2} \mathrm{C}_{2} \mathrm{~S}_{2}\right) \mathrm{Ni}\left(\mathrm{tpbzO}_{2}\right)\right], \quad\left[\left(\mathrm{Me}_{2} \mathrm{C}_{2} \mathrm{~S}_{2}\right) \mathrm{Pt}\right.$ $\left(\right.$ tpbzO $\left.\left.\mathrm{O}_{2}\right)\right]$, and $\left[\left(\mathrm{Me}_{2} \mathrm{C}_{2} \mathrm{~S}_{2}\right) \mathrm{Ni}\left(\mathrm{tpbzS}_{2}\right)\right]$-have been prepared in a straightforward fashion using $\mathrm{I}_{2} /$ air, $\mathrm{H}_{2} \mathrm{O}_{2},\left[\mathrm{NH}_{4}\right]_{2}[\mathrm{~S}]$,
Table 6. Selected Interatomic Distances (Å), Bond Angles (deg), and Other Structural Parameters for $16-18^{a}$

$\begin{array}{llll} & {\left[\left(\mathrm{Ph}_{2} \mathrm{C}_{2} \mathrm{~S}_{2}\right)\right.} & {\left[\left(\mathrm{Me}_{2} \mathrm{C}_{2} \mathrm{~S}_{2}\right)\right.} & {\left[\left(\mathrm{Me}_{2} \mathrm{C}_{2} \mathrm{~S}_{2}\right)\right.} \\ \left.\mathrm{Ni}\left(\mathrm{tpbzO}_{2}\right)\right] & \begin{array}{l}\left.\mathrm{Pt}\left(\mathrm{tpbzO}_{2}\right)\right] \\ \left.\mathrm{Ni}\left(\mathrm{tpbz} \mathrm{S}_{2}\right)\right]\end{array} \\ \mathrm{M}-\mathrm{S} & 2.1477[5] & 2.2934[4] & 2.137[1] \\ \mathrm{M}-\mathrm{P} & 2.1656[5] & 2.2588[3] & 2.141[1] \\ \Delta^{b} & +0.0179 & -0.0346 & +0.004 \\ \mathrm{~S}-\mathrm{C} & 1.759[1] & 1.759[1] & 1.755[6] \\ \mathrm{P}-\mathrm{C}^{c} & 1.833[1] & 1.8247[12] & 1.836[5] \\ \mathrm{P}-\mathrm{C}^{d} & 1.840[1] & 1.8323[12] & 1.848[5] \\ \mathrm{P}=\mathrm{E} & 1.4931[12] & 1.4936[11] & 1.929[2] \\ \mathrm{S}-\mathrm{M}-\mathrm{S} & 90.86(2) & 88.006(18) & 92.13(9) \\ \mathrm{P}-\mathrm{M}-\mathrm{P} & 89.42(2) & 86.717(16) & 89.95(8) \\ \mathrm{S}-\mathrm{M}-\mathrm{P}_{\text {cis }} & 89.83[1] & 93.265[12] & 91.06[6] \\ \mathrm{S}-\mathrm{M}-\mathrm{P}_{\text {trans }} & 177.76[2] & 171.307[12] & 164.34[6] \\ \theta,{ }^{e} \text { deg } & 2.3 & 12.3 & 22.1 \\ \varphi^{f} \text { deg } & 5.5 & 19.1 & 7.9 \\ \text { Averaged } & & & \end{array}$

${ }^{a}$ Averaged values are given where two or more chemically identical interatomic distances or angles are present. Uncertainties are propagated according to Taylor, J. R. An Introduction to Error Analysis, 2nd ed.; University Science Books: Sausalito, CA, 1997; pp 73-77; propagated uncertainties are designated with [ ]. ${ }^{b} \Delta=(\mathrm{M}-$ $\mathrm{P})-(\mathrm{M}-\mathrm{S})$ bond length difference. ${ }^{c} \mathrm{C}$ atom of the central arene ring, metalated side. ${ }^{d} \mathrm{C}$ atom of the central arene ring, open side. ${ }^{e}$ Angle between the $\mathrm{S}_{2} \mathrm{M}$ and $\mathrm{P}_{2} \mathrm{M}$ planes. ${ }^{f}$ Angle between the $\mathrm{MP}_{2}$ plane and $\mathrm{P}_{2} \mathrm{C}_{6} \mathrm{P}_{2}$ mean plane. 
Scheme 4. Preparation of $\left[\mathrm{Ni}\left(\mathrm{dppbO}_{2}\right)_{3}\right]\left[\mathrm{I}_{3}\right]_{2}$

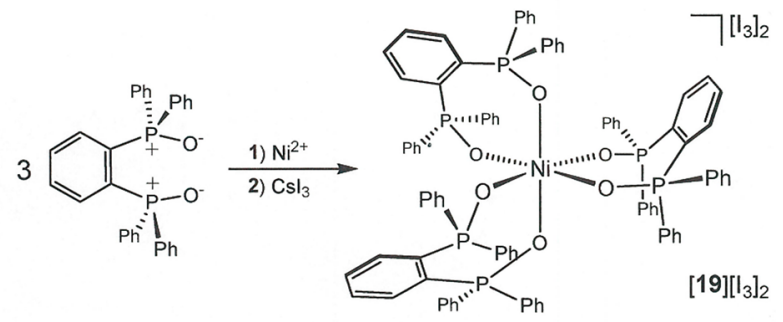

respectively. For reasons not immediately obvious, the known $\mathrm{I}_{2} /$ air protocol $^{11}$ that works to produce $\mathbf{1 6}$ in modest yield appears to be ineffective in generating the Pt compound 17 . However, the more vigorous $\mathrm{H}_{2} \mathrm{O}_{2}$ effectively oxidizes 7 to afford 17 in a yield comparable to the production of $\mathbf{1 6}$ from 8 by $\mathrm{I}_{2} /$ air. Considering the reported susceptibility of [(bdt)Pt(bipy)] to complete oxidation of the thiolate $S$ atoms to the disulfinate form in the presence of light and $\mathrm{O}_{2}{ }^{22}$ it is somewhat surprising that the often indiscriminate $\mathrm{H}_{2} \mathrm{O}_{2}$ can produce 17 without significant attending decomposition. These several oxidized compounds are readily distinguished by ${ }^{31} \mathrm{P}$ NMR spectroscopy from the open-ended $\left[\left(\mathrm{R}_{2} \mathrm{C}_{2} \mathrm{~S}_{2}\right)\right.$ $\mathrm{M}(\mathrm{tpbz})$ ] compounds because the signal arising from the trivalent $P$ is moved $40-60 \mathrm{ppm}$ downfield and nearer to the signal arising from the $\mathrm{P}$ nuclei bound to $\mathrm{M}^{2+}$. Structurally, 16-18 are similar to 5-9 and $\mathbf{1 1}$ in having closer adherence to planarity for the heavier metal, positive and negative $\Delta$ (Table 6) for the $\mathrm{Ni}$ and $\mathrm{Pt}$ compounds, respectively, and modestly longer $\mathrm{P}-\mathrm{C}_{\text {central arene }}$ bond lengths at the open end versus the metalated end. The bond lengths from $\mathrm{P}$ to terminal chalogenide are typically at $\sim 1.49 \AA$ for the oxide ${ }^{23,24}$ and $\sim 1.93 \AA$ for the sulfide. ${ }^{25,26}$

The potential utility of compounds such as 17 and 18 is: (1) Their capacity to select different ions at their open end versus $\left[\left(\mathrm{R}_{2} \mathrm{C}_{2} \mathrm{~S}_{2}\right) \mathrm{M}(\mathrm{tpbz})\right]$ by virtue of the ylide character to the phosphine sulfide or oxide; (2) their ability to form homoleptic tris(chelate) complexes because the otherwise prohibitive congestion that would be occasioned by six $\mathrm{Ph}_{2} \mathrm{P}$ groups is further removed from the coordination sphere of $\mathrm{M}^{n+}$. Such possibilities are intimated by the finding that a test reaction between the related $\mathrm{dppbO}_{2}$ and $\mathrm{Ni}^{2+}$ leads to octahedral $\left[\left(\mathrm{dppbO}_{2}\right)_{3} \mathrm{Ni}\right]^{2+}$, which has been isolated as its $\mathrm{I}_{3}^{-}$salt (Scheme 4 and Figure 3) in both triclinic and monoclinic forms. Selected structural parameters, which are highly similar for both polymorphs, are presented in Table S5. The phosphoryl P-O bond lengths in $[19]^{2+}(1.491[1] \AA)$ are slightly longer than those in the free ligand $(1.485[1] \AA){ }^{24}$ Each ligand shows a substantial folding around the intraligand $\mathrm{O} \cdots \mathrm{O}$ axis such that the $\mathrm{NiO}_{2}$ plane meets the $\mathrm{P}_{2} \mathrm{C}_{2}$ chelate mean plane at an angle of $\sim 57^{\circ}$ (average of six values). For both structures of $[19]^{2+}$, the directionality of this folding is different for one $\mathrm{dppbO}_{2}$ ligand than it is for the other two, thus lowering the symmetry from $C_{3}$ to $C_{1}$. These structures for $[19]^{2+}$ appear to be the first for a homoleptic tris(chelate) coordination complex with $\mathrm{dppbO}_{2}$, although a preparation of the $\left[\left(\mathrm{dppbO}_{2}\right)_{3} \mathrm{M}\right]^{2+}\left(\mathrm{M}=\mathrm{Mg}^{2+}, \mathrm{Ca}^{2+}, \mathrm{Sr}^{2+}, \mathrm{Ba}^{2+}\right)$ series has been reported. ${ }^{27}$ A related tris(chelate) of $\mathrm{Ni}^{2+}$, $\left[\left(\text { dppeO }_{2}\right)_{3} \mathrm{Ni}\right]^{2+}\left(\right.$ dppeO$_{2}=1,2$-bis (diphenylphosphino $)-$ ethane dioxide), has been structurally authenticated ${ }^{28}$ but notably contrasts with $[19]^{2+}$ in having a much more modest average angle of $9.2^{\circ}$ between the $\mathrm{NiO}_{2}$ and $\mathrm{P}_{2} \mathrm{C}_{2}$ chelate planes for its ligands.

Electrochemistry. The open-ended compounds $\left[\left(\mathrm{R}_{2} \mathrm{C}_{2} \mathrm{~S}_{2}\right) \mathrm{M}(\mathrm{tpbz})\right]$ generally support a reversible $1 \mathrm{e}^{-}$ oxidation that is attributed to transformation of the ene-1,2dithiolate dianion into its radical monoanionic form (Scheme 1 , a $\rightarrow \mathbf{b}$ ). The potential at which this oxidation occurs is shifted cathodically by $\sim 0.050 \mathrm{~V}$ compared to the corresponding feature in the symmetric, dimetallic complex. This modest shift appears to be due to an inherent electron-donating character of the tetraphosphino ligand platform, which is tempered somewhat when the ligand's open end chelates a second $\mathrm{M}\left(\mathrm{S}_{2} \mathrm{C}_{2} \mathrm{R}_{2}\right)$ group. However, the second reversible oxidation that occurs in $\left[\left(\mathrm{R}_{2} \mathrm{C}_{2} \mathrm{~S}_{2}\right) \mathrm{M}(\mathrm{tpbz}) \mathrm{M}\left(\mathrm{S}_{2} \mathrm{C}_{2} \mathrm{R}_{2}\right)\right](\mathrm{M}=$ $\mathrm{Ni}, \mathrm{Pd}, \mathrm{Pt} ; \mathrm{R}=\mathrm{Ph}, \mathrm{p}$-anisyl) is typically irreversible in their open-ended counterparts (Table 7$)$. In $\left[\left(\mathrm{R}_{2} \mathrm{C}_{2} \mathrm{~S}_{2}\right) \mathrm{M}(\mathrm{tpbz}) \mathrm{M}\right.$ $\left(\mathrm{S}_{2} \mathrm{C}_{2} \mathrm{R}_{2}\right)$, this second wave was assigned to radical monoanion to $\alpha$-dithione oxidation (Scheme $1, \mathbf{b} \rightarrow \mathbf{c}$ ). Assuming a similar description pertains to the open-ended

Table 7. Electrochemical Data (V) for the Open-Ended Compounds 4-6 and 8-15 for Dimetallic tpbz-Bridged 15 and for Open-Ended Oxidized 16 versus $\mathrm{Cp}_{2} \mathrm{Fe}^{+} / \mathrm{Cp}_{2} \mathrm{Fe}$ with a $0.10 \mathrm{M}\left[\mathrm{Bu}_{4} \mathrm{~N}\right]\left[\mathrm{PF}_{6}\right]$ Supporting Electrolyte, a Glassy Carbon or Pt Disk Working Electrode, and $\mathrm{CH}_{2} \mathrm{Cl}_{2}$ as the Solvent ${ }^{a}$

\begin{tabular}{|c|c|c|c|c|c|}
\hline & $E_{4}(\mathrm{ox})$ & $E_{3}($ ox $)$ & $E_{2}($ ox $)$ & $E_{1}($ ox $)$ & $E_{1}(\mathrm{red})$ \\
\hline$[(\mathrm{mnt}) \mathrm{Ni}(\mathrm{tpbz})]^{b}, \mathbf{4}$ & & & & $+0.62(\mathrm{ir}, 1 \mathrm{e})^{d}$ & $-1.53(\mathrm{r}, 1 \mathrm{e})^{e}$ \\
\hline$[(\mathrm{mdt}) \mathrm{Ni}(\mathrm{tpbz})]^{c}, \mathbf{5}$ & & & $+0.71(\mathrm{ir}, 1 \mathrm{e})^{d}$ & $0.00(\mathrm{r}, 1 \mathrm{e})^{e}$ & $-2.11(\mathrm{qr}, 1 \mathrm{e})^{f}$ \\
\hline$[(\mathrm{mdt}) \operatorname{Pt}(\mathrm{tpbz})]^{c}, \mathbf{6}$ & & $+0.85(\mathrm{ir})^{g}$ & $+0.66(\text { ir })^{g}$ & $-0.01(\mathrm{r}, 1 \mathrm{e})^{e}$ & \\
\hline$[(\mathrm{pdt}) \mathrm{Ni}(\mathrm{tpbz})]^{c}, \mathbf{8}$ & & & $+0.71(\mathrm{ir}, 1 \mathrm{e})^{e}$ & $+0.09(\mathrm{r}, 1 \mathrm{e})^{e}$ & $-2.10(\mathrm{r}, 1 \mathrm{e})^{e}$ \\
\hline$[(\mathrm{pdt}) \operatorname{Pd}(\mathrm{tpbz})]^{c}, 9$ & & $+0.85(\mathrm{ir}, 1 \mathrm{e})^{d}$ & $\sim+0.66(\text { ir, } 1 \mathrm{e})^{d}$ & $+0.05(\mathrm{r}, 1 \mathrm{e})^{e}$ & \\
\hline$[(\mathrm{pdt}) \operatorname{Pt}(\mathrm{tpbz})]^{c}, \mathbf{1} \mathbf{1}$ & & $+0.88(\mathrm{qr}, 1 \mathrm{e})^{e}$ & $+0.54(\mathrm{ir}, 1 \mathrm{e})^{d}$ & $+0.13(\mathrm{r}, 1 \mathrm{e})^{e}$ & $-1.97(\mathrm{qr}, 1 \mathrm{e})^{f}$ \\
\hline$\left[(\mathrm{pdt})_{2} \mathrm{Pt}(\mathrm{tpbz})\right]^{b}, \mathbf{1 0}$ & $+0.66(\mathrm{ir})^{g}$ & $+0.48(\mathrm{r}, 1 \mathrm{e})^{e}$ & $+0.16(\mathrm{qr}, 1 \mathrm{e})^{f}$ & $-0.02(\mathrm{r}, 1 \mathrm{e})^{e}$ & $-1.22(\text { ir })^{g}$ \\
\hline$[(\mathrm{adt}) \mathrm{Ni}(\mathrm{tpbz})]^{c}, \mathbf{1 2}$ & & & $+0.59(\mathrm{qr}, 1 \mathrm{e})^{f}$ & $+0.02(\mathrm{r}, 1 \mathrm{e})^{e}$ & $-2.11(\mathrm{qr}, 1 \mathrm{e})^{f}$ \\
\hline$[(\mathrm{adt}) \operatorname{Pd}(\mathrm{tpbz})]^{c}, \mathbf{1 3}$ & & & $+0.67(\mathrm{ir}, 1 \mathrm{e})^{d}$ & $+0.09(\mathrm{r}, 1 \mathrm{e})^{e}$ & \\
\hline$[(\mathrm{adt}) \mathrm{Pt}(\mathrm{tpbz})]^{c}, \mathbf{1 4}$ & & & $+0.60(\mathrm{qr}, 1 \mathrm{e})^{f}$ & $+0.01(\mathrm{r}, 1 \mathrm{e})^{e}$ & \\
\hline$[(\mathrm{pdt}) \operatorname{Pt}(\mathrm{tpbz}) \mathrm{Ni}(\mathrm{mdt})]^{c}, \mathbf{1 5}$ & $+0.92(\mathrm{r}, 1 \mathrm{e})^{e}$ & $+0.70(\mathrm{r}, 1 \mathrm{e})^{e}$ & $+0.19(\mathrm{r}, 1 \mathrm{e})^{e}$ & $+0.06(\mathrm{r}, 1 \mathrm{e})^{e}$ & $-1.97(\mathrm{r}, 1 \mathrm{e})^{e}$ \\
\hline$\left[(\mathrm{pdt}) \mathrm{Ni}\left(\mathrm{tpbzO}_{2}\right)\right]^{c}, \mathbf{1 6}$ & & & $+0.75(\mathrm{qr}, 1 \mathrm{e})^{f}$ & $0.12(\mathrm{r}, 1 \mathrm{e})^{e}$ & $-1.98(\mathrm{r}, 1 \mathrm{e})^{e}$ \\
\hline
\end{tabular}

${ }^{a}$ Ligand abbreviations: $\mathrm{mnt}=\left[(\mathrm{NC})_{2} \mathrm{C}_{2} \mathrm{~S}_{2}\right]^{2-}$, mdt $=\left[\mathrm{Me}_{2} \mathrm{C}_{2} \mathrm{~S}_{2}\right]^{2-}$, pdt $=\left[\mathrm{Ph}_{2} \mathrm{C}_{2} \mathrm{~S}_{2}\right]^{2-}$, and adt $=\left[\left(\mathrm{MeO}-p-\mathrm{C}_{6} \mathrm{H}_{4}\right)_{2} \mathrm{C}_{2} \mathrm{~S}_{2}\right]^{2-}$. ${ }^{b} \mathrm{~A} \mathrm{Pt}$ disk working electrode was used. ${ }^{c} \mathrm{~A}$ glassy carbon working electrode was used. ${ }$ ir $=$ irreversible; value obtained by differential pulse voltammetry. ${ }^{e} \mathrm{r}=$ reversible; value obtained by cyclic voltammetry. $f_{\mathrm{qr}}=$ quasireversible; value obtained by cyclic voltammetry. ${ }_{\mathrm{ir}}=$ irreversible; value estimated from the anodic maximum, $E_{\mathrm{a}}$, in the cyclic voltammogram. 


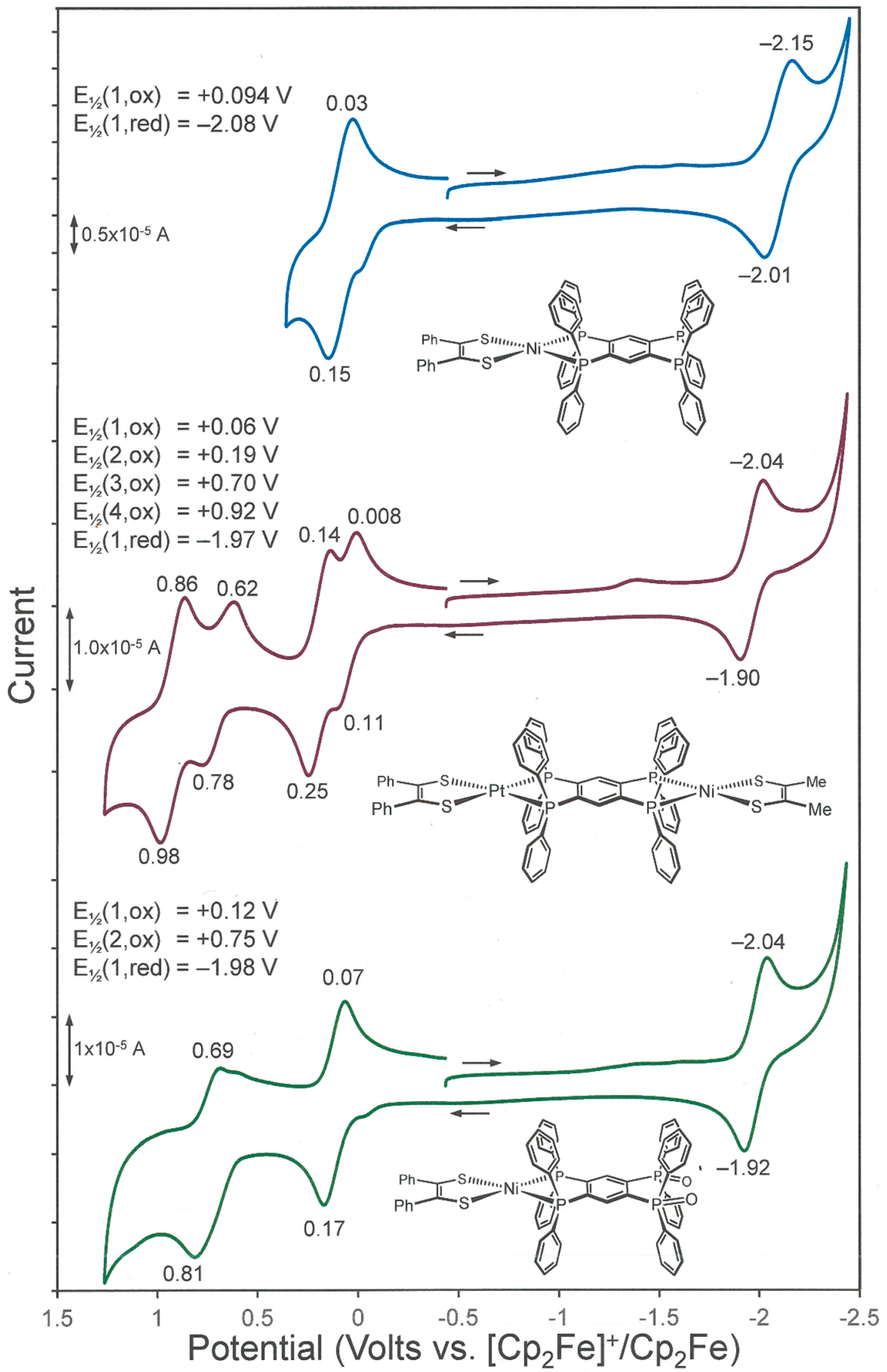

Figure 4. Cyclic voltammetry of selected compounds from Schemes 2 and 3.

compounds, it is unclear why reversible behavior is not sustained under the same conditions. The cathodic direction reveals a single $1 \mathrm{e}^{-}$reduction for $\left[\left(\mathrm{R}_{2} \mathrm{C}_{2} \mathrm{~S}_{2}\right) \mathrm{M}(\mathrm{tpbz})\right]$ that is due to a reduction of the tpbz ligand. The more comparable scaling of the current amplitude for this reduction relative to the oxidation waves is consistent with the assignment of all processes as one-electron events (Figure 4 (top)). In contrast, the oxidation waves in $\left[\left(\mathrm{R}_{2} \mathrm{C}_{2} \mathrm{~S}_{2}\right) \mathrm{M}(\mathrm{tpbz}) \mathrm{M}\left(\mathrm{S}_{2} \mathrm{C}_{2} \mathrm{R}_{2}\right)\right]$ are $2 \mathrm{e}^{-}$ processes and display substantially greater current compared to the reduction wave, which is also due to the reduction of tpbz. 


\section{Highest Occupied MOs for [(pdt)Pt(tpbz)Ni(mdt)]}

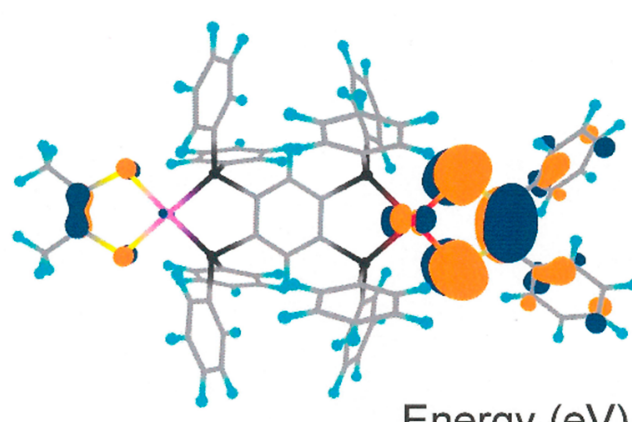

HOMO-1

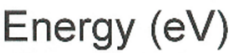

Relative to HOMO
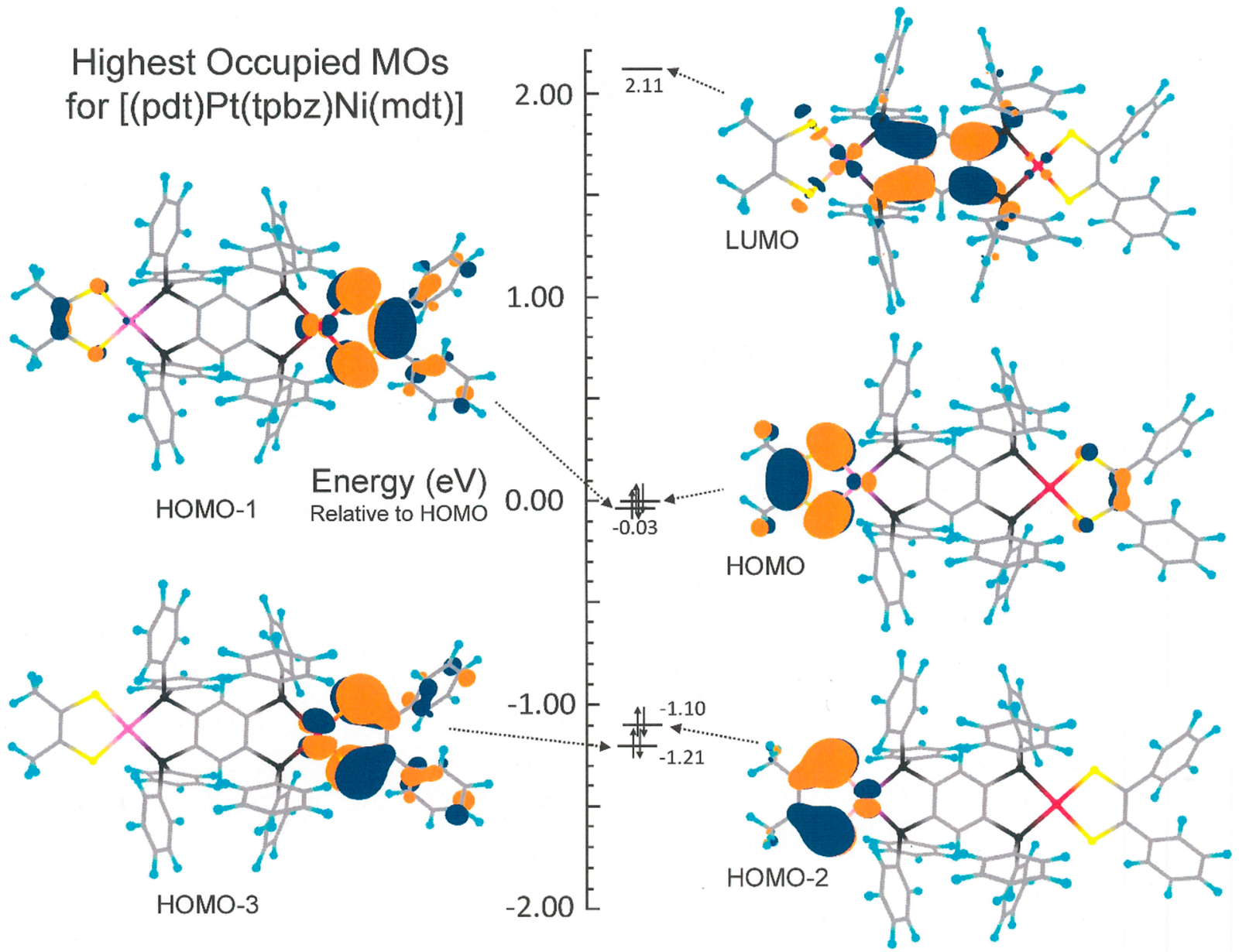

Figure 5. Frontier molecular orbitals for $\left[\left(\mathrm{Ph}_{2} \mathrm{C}_{2} \mathrm{~S}_{2}\right) \mathrm{Pt}(\mathrm{tpbz}) \mathrm{Ni}\left(\mathrm{S}_{2} \mathrm{C}_{2} \mathrm{Me}_{2}\right)\right]$, showing the HOMO and HOMO-1 to be largely localized at the $\mathrm{Ni}\left(\mathrm{S}_{2} \mathrm{C}_{2} \mathrm{Me}_{2}\right)$ and $\mathrm{Pt}\left(\mathrm{S}_{2} \mathrm{C}_{2} \mathrm{Ph}_{2}\right)$ ends, respectively. Orbital images are shown at the 0.03 contour level.

In open-ended $\left[\left(\mathrm{R}_{2} \mathrm{C}_{2} \mathrm{~S}_{2}\right) \mathrm{M}(\mathrm{tpbz})\right]$, the reduction wave is shifted to a more negative potential than that in the corresponding dimetallic compound. Here again, the effect is accounted for by the greater electron richness of the tpbz ligand when metalated at one end instead of both. The description of these redox events is corroborated by structure optimizations that reveal the HOMO and LUMO for 8 to be dithiolene-based and predominantly tpbz-based, respectively (Figure S116).

Because of the asymmetry in 15 , the successive oxidation waves that arise from ene-1,2-dithiolate to radical monoanion (Scheme 1, a $\rightarrow \mathbf{b}$ ) and radical monoanion to $\alpha$-dithione (Scheme $1, \mathbf{b} \rightarrow \mathbf{c}$ ) oxidation are partially resolved [Figure 4 (middle)]. In our earlier study involving the centrosymmetric homodimetallic compounds $\left[\left(\mathrm{R}_{2} \mathrm{C}_{2} \mathrm{~S}_{2}\right) \mathrm{M}(\mathrm{tpbz}) \mathrm{M}\left(\mathrm{S}_{2} \mathrm{C}_{2} \mathrm{R}_{2}\right)\right]$, a consistent anodic shift of $+0.04-0.05 \mathrm{~V}$ was observed upon moving from the methyl- to phenyl-substituted dithiolene ligand, while the metal ion was constant as either $\mathrm{Ni}^{2+}, \mathrm{Pd}^{2+}$, or $\mathrm{Pt}^{2+}$. This difference was attributed to a modestly greater electron-donating effect for $\mathrm{Me}$ over $\mathrm{Ph}$, which enables the ligand-based oxidation to occur at a less positive potential. Similarly, the change in $\left[\left(\mathrm{R}_{2} \mathrm{C}_{2} \mathrm{~S}_{2}\right) \mathrm{M}(\mathrm{tpbz}) \mathrm{M}\left(\mathrm{S}_{2} \mathrm{C}_{2} \mathrm{R}_{2}\right)\right]$ from $\mathrm{Ni}^{2+}$ to $\mathrm{Pt}^{2+}$ with a constant dithiolene ligand occasioned a $+0.13-0.14 \mathrm{~V}$ anodic shift, which was ascribed to a greater dipositive character of the third-row metal compared to the first-row metal. Assuming the effects of the dithiolene substituent and of the metal-ion identity to be simply additive leads to the prediction that $\left[\left(\mathrm{Ph}_{2} \mathrm{C}_{2} \mathrm{~S}_{2}\right) \mathrm{Pt}(\mathrm{tpbz}) \mathrm{Ni}\left(\mathrm{S}_{2} \mathrm{C}_{2} \mathrm{Me}_{2}\right)\right]$ would undergo oxidation at its $\mathrm{Ni}\left(\mathrm{S}_{2} \mathrm{C}_{2} \mathrm{Me}_{2}\right)$ end first, with $\sim 0.18 \mathrm{~V}$ separating that process from an oxidation of the same nature at the $\operatorname{Pt}\left(\mathrm{S}_{2} \mathrm{C}_{2} \mathrm{Ph}_{2}\right)$ end. As gauged by the anodic and cathodic maxima in the voltammogram [Figure 4 (middle)], the $\Delta E_{1 / 2}$ separating the first and second oxidation processes is $\sim 0.13 \mathrm{~V}$, while the $\Delta E_{1 / 2}$ between the third and fourth waves is $\sim 0.22 \mathrm{~V}$. The assignment of the first anodic wave as $\mathrm{Ni}\left({ }^{-} \mathrm{S}_{2} \mathrm{C}_{2} \mathrm{Me}_{2}\right)-1 \mathrm{e}^{-} \rightarrow \mathrm{Ni}\left({ }^{-} \mathrm{S}^{\bullet} \mathrm{SC}_{2} \mathrm{Me}_{2}\right)^{+}$and the second as $\left(\mathrm{Ph}_{2} \mathrm{C}_{2} \mathrm{~S}_{2}^{-}\right) \mathrm{Pt}-1 \mathrm{e}^{-} \rightarrow\left(\mathrm{Ph}_{2} \mathrm{C}_{2} \mathrm{~S}^{-} \mathrm{S}^{\bullet}\right) \mathrm{Pt}^{+}$is affirmed by a geometry optimization of 15 . The calculated electronic structure shows the HOMO to be predominantly constituted of the $\mathrm{Ni}\left(\mathrm{S}_{2} \mathrm{C}_{2} \mathrm{Me}_{2}\right)$ end, while the $\mathrm{HOMO}-1$, which likely becomes the HOMO in $[\mathbf{1 5}]^{+}$, is largely localized at the opposite $\left(\mathrm{Ph}_{2} \mathrm{C}_{2} \mathrm{~S}_{2}\right) \mathrm{Pt}$ terminus (Figure 5$)$. The $1 \mathrm{e}^{-}$cathodic wave at $-1.97 \mathrm{~V}$, which arises from a reduction of the tpbz ligand, occurs at a potential very similar to its place in the homodimetallic compounds.

Although they have been known for some time, $, 21,29-31$ platinum dithiolene compounds of the type $\left[\left(\mathrm{R}_{2} \mathrm{C}_{2} \mathrm{~S}_{2}\right)_{2} \mathrm{Pt}\right.$ (phosphine $\left.)_{2}\right]$ have been little studied electrochemically. In the oxidizing direction, the cyclic voltammogram of $\mathbf{1 0}$ reveals multiple reversible and partially reversible waves (Figure S75). Given the reduced state of the dithiolene ligand, as inferred from the structural data, these processes are likely successive $1 \mathrm{e}^{-}$oxidations of the dithiolene ligands, of which there are four in principle. Cathodic scanning reveals irreversible behavior 


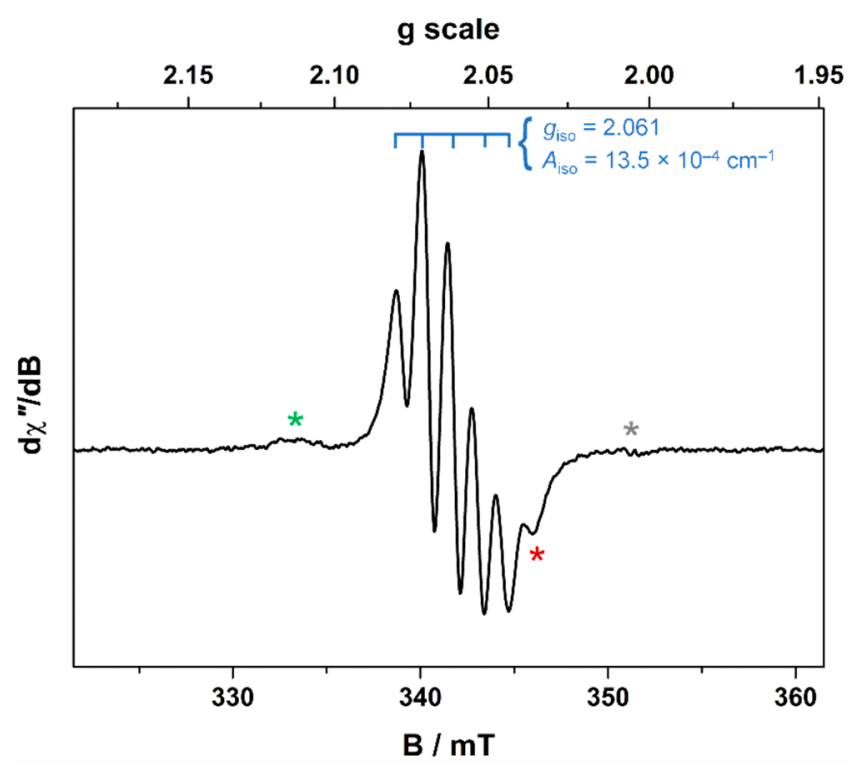

Figure 6. X-band EPR spectrum of 8 treated with 2 equiv of tris(4bromophenyl)ammoniumyl hexachloroantimonate in $\mathrm{CH}_{2} \mathrm{Cl}_{2}$ recorded at room temperature (experimental conditions: frequency, $9.8587 \mathrm{GHz}$; power, $6.3 \mathrm{~mW}$; modulation, $0.4 \mathrm{mT}$ ). The main signal indicated in blue comprises the five lines with $g_{\text {iso }}=2.061$ and $A_{\text {iso }}=$ $13.5 \times 10^{-4} \mathrm{~cm}^{-1}$. Minor signals are denoted with the red and green asterisks. The gray asterisk references the position of the oxidant $(g \sim$ 2.0036).

beginning at $\sim-1.22 \mathrm{~V}$ versus the $\left[\mathrm{Cp}_{2} \mathrm{Fe}\right]^{+} /\left[\mathrm{Cp}_{2} \mathrm{Fe}\right]$ couple, which is likely metal-based and possibly leads to the extrusion of one of the dithiolene ligands.

EPR Spectroscopy. An aliquot of $\mathbf{8}$ was treated with successive equivalents of tris(4-bromophenyl)ammoniumyl hexachloroantimonate and monitored by EPR spectroscopy. The addition of 0.5 and 1 equiv of oxidant yielded no signal, although the reduction potential of the oxidant $0.7 \mathrm{~V}\left(\mathrm{vs} \mathrm{Fc}^{+/ 0}\right.$ ) is sufficient to oxidize this compound. ${ }^{32}$ At $>1$ equiv, the main signal shown in the spectrum in Figure 6 appeared, which is comprised of fives lines in a binomial intensity pattern brought about by the coupling of four ${ }^{31} \mathrm{P}$ nuclei. The signal is shortlived, diminishing over the course of $2-3 \mathrm{~h}$ even in a Teflonstoppered EPR tube. On the basis of its relative intensity and position, the feature denoted by the red asterisk is a separate signal, presumably the high-field hyperfine component of a multiline signal that lies beneath the main signal. A second minor signal is identified at $g \sim 2.011$, as indicated by the green asterisk, and comprises a weakly resolved three-line binomial pattern.

At this stage, no definitive assignment can be made of the EPR signal. The $g$ and $A$ values are distinct from other paramagnetic nickel diphosphine species. For example, the spin doublet $\left[\left(\mathrm{adt}^{\bullet}\right) \mathrm{Ni}^{\mathrm{II}}(\mathrm{dppb})\right]^{+}$has a three-line spectrum with $g_{\text {iso }}$ $=2.0106$ and $A_{\text {iso }}=3.3 \times 10^{-4} \mathrm{~cm}^{-1}$, while the spin triplet $\left[\left(\mathrm{adt}^{\bullet}\right) \mathrm{Ni}^{\mathrm{II}}(\mathrm{tpbz}) \mathrm{Ni}^{\mathrm{II}}\left(\mathrm{adt}^{\bullet}\right)\right]^{+}$has a five-line signal with $g_{\text {iso }}=$ 2.011 and $A_{\text {iso }}=1.7 \times 10^{-4} \mathrm{~cm}^{-1}$ [where $\operatorname{adt}^{2-}=\operatorname{bis}(p$-anisyl)1,2-ethenedithiolate.$^{33}$ The hyperfine coupling constant is $25 \%$ of that in the spectrum in Figure 6 . The $g$ values are also noticeably smaller, where the higher value here is better matched by $\left[\mathrm{Ni}(\mathrm{adt})\left(\mathrm{adt}^{\bullet}\right)\right]^{-}$with $g_{\text {iso }}=2.0059$ (but with no hyperfine splitting). In contrast, Ni-centered $S=1 / 2$ complexes with phosphine ligands have even larger hyperfine coupling constants, e.g., $\left[\mathrm{Ni}^{\mathrm{I}}(\mathrm{dppe})_{2}\right]^{+}$with $g_{\text {iso }}=2.090$ and $A_{\text {iso }}=83 \times$
$10^{-4} \mathrm{~cm}^{-1}$ and $\left[\left(\mathrm{Et}_{2} \mathrm{dtc}\right) \mathrm{Ni}^{\mathrm{I}}(\mathrm{dppe})\right]^{0}$ with $g_{\text {iso }}=2.089$ and $A_{\text {iso }}$ $=81 \times 10^{-4} \mathrm{~cm}^{-1}$ [dppe $=1,2$-bis (diphenylphosphino)ethane; $\mathrm{Et}_{2} \mathrm{dtc}=\mathrm{N}, \mathrm{N}$-diethyldithiocarbamate $] .{ }^{30}$ The main signal here is consistent with four $\mathrm{P}$ donor atoms at a $\mathrm{Ni}^{\mathrm{II}}$ center with a coordinated dithiolene radical and results from subsequent oxidation of the first oxidation product of the monometallic $[(\mathrm{pdt}) \mathrm{Ni}(\mathrm{tpbz})]$. The exact composition and construct of this paramagnetic species are not known at this time.

\section{SUMMARY AND CONCLUSIONS}

The principal findings of this work are the follows:

(1) Open-ended compounds of the type $\left[\left(\mathrm{R}_{2} \mathrm{C}_{2} \mathrm{~S}_{2}\right) \mathrm{M}\right.$ $(\mathrm{tpbz})]$ can be prepared in good yields either by the direct reaction between charge-neutral $\left[\left(\mathrm{R}_{2} \mathrm{C}_{2} \mathrm{~S}_{2}\right)_{2} \mathrm{M}\right]\left(\mathrm{M}=\mathrm{Ni}^{2+}\right.$, $\mathrm{Pd}^{2+}, \mathrm{Pt}^{2+} ; \mathrm{R}=\mathrm{Me}, \mathrm{Ph}, p$-anisyl) and tpbz or by transmetalation between $\left[\mathrm{Cl}_{2} \mathrm{M}(\mathrm{tpbz})\right]$ and $\left[\left(\mathrm{R}_{2} \mathrm{C}_{2} \mathrm{~S}_{2}\right) \mathrm{SnR}_{2}^{\prime}\right]$ $\left(\mathrm{R}=\mathrm{Me}, \mathrm{R}^{\prime}={ }^{n} \mathrm{Bu} ; \mathrm{R}=\mathrm{CN}, \mathrm{R}^{\prime}=\mathrm{Me}\right)$.

(2) The open-ended $\left[\left(\mathrm{R}_{2} \mathrm{C}_{2} \mathrm{~S}_{2}\right) \mathrm{M}(\mathrm{tpbz})\right]$ compounds reveal ${ }^{31} \mathrm{P}$ NMR signals in the $55-40 \mathrm{ppm}$ range and at $\sim-15.0 \mathrm{ppm}$, corresponding respectively to the metalated and open ends, in clear distinction from their symmetric homodimetallic counterparts.

(3) The open-ended compounds are subject to the reaction types typical of chelating diphosphines. They may be oxidized to diphosphine dicalcogenides at their open end, and they may be metalated asymmetrically with an altogether different $\mathrm{ML}_{n}$ fragment.

(4) The cyclic voltammogram of the asymmetric heterodimetallic compound $\left[\left(\mathrm{Ph}_{2} \mathrm{C}_{2} \mathrm{~S}_{2}\right) \mathrm{Pt}(\mathrm{tpbz}) \mathrm{Ni}\left(\mathrm{S}_{2} \mathrm{C}_{2} \mathrm{Me}_{2}\right)\right]$ shows two pairs of closely spaced, but resolved, $1 \mathrm{e}^{-}$oxidations that correspond to the successive oxidation of each metallodithiolene end group, first to the radical monoanion state and then to the $\alpha$-dithione. In contrast, the centrosymmetric homodimetallic $\left[\left(\mathrm{R}_{2} \mathrm{C}_{2} \mathrm{~S}_{2}\right) \mathrm{M}(\mathrm{tpbz}) \mathrm{M}\left(\mathrm{S}_{2} \mathrm{C}_{2} \mathrm{R}_{2}\right)\right]$ compounds show two $2 \mathrm{e}^{-}$oxidation waves.

In forthcoming reports, we detail the syntheses, structures, and properties of heterotrimetallic compounds of the form $\left[\left[\left(\mathrm{R}_{2} \mathrm{C}_{2} \mathrm{~S}_{2}\right) \mathrm{M}(\mu \text {-tpbz })\right]_{2} \mathrm{M}^{\prime} \mathrm{L}_{x}\right]^{n}$, where $\mathrm{M}^{\prime} \mathrm{L}_{x}$ is a either a charge-neutral fragment or a cation, using the monometallic $\left[\left(\mathrm{R}_{2} \mathrm{C}_{2} \mathrm{~S}_{2}\right) \mathrm{M}(\mathrm{tpbz})\right]$ complexes described here as fungible building units.

\section{ASSOCIATED CONTENT}

\section{sI Supporting Information}

The Supporting Information is available free of charge at https://pubs.acs.org/doi/10.1021/acs.inorgchem.1c01573.

Procedures for crystal growth, X-ray diffraction data collection, and structure solution and refinement, summaries of the unit cell and refinement data (Tables S1-S4), thermal ellipsoid plots with complete atom labeling (Figures S1-S23), spectroscopic, electrochemical, and analytical data for the compounds reported (Figures S24-S115), a description of the computational procedures, an MO energy level diagram for 8 (Figure S116), and coordinates for geometry-optimized 15 and 8 (Tables S6 and S7) (PDF)

\section{Accession Codes}

CCDC 2024446-2024456 and 2076132-2076135 contain the supplementary crystallographic data for this paper. These data can be obtained free of charge via www.ccdc.cam.ac.uk/ data_request/cif, or by emailing data_request@ccdc.cam.ac. uk, or by contacting The Cambridge Crystallographic Data 
Centre, 12 Union Road, Cambridge CB2 1EZ, UK; fax: +44 1223336033.

\section{AUTHOR INFORMATION}

\section{Corresponding Authors}

Satyendra Kumar - Department of Chemistry, Tulane University, New Orleans, Louisiana 70118, United States; Present Address: Xavier University of Louisiana, 1 Drexel Drive, New Orleans, Louisiana 70125; Email: skumar5@ tulane.edu

James P. Donahue - Department of Chemistry, Tulane University, New Orleans, Louisiana 70118, United States; 다이.org/0000-0001-9768-4813; Email: donahue@ tulane.edu

\section{Authors}

Malathy Selvachandran - Department of Chemistry, Tulane University, New Orleans, Louisiana 70118, United States; Present Address: University of Jaffna, Vavuniya Campus, Jaffna, Sri Lanka.

Kuppuswamy Arumugam - Department of Chemistry, Wright State University, Dayton, Ohio 45435-0001, United States; ○ orcid.org/0000-0003-2654-5001

Mohamed C. Shaw - Department of Chemistry, Tulane University, New Orleans, Louisiana 70118, United States

Che Wu - Department of Chemistry, Tulane University, New Orleans, Louisiana 70118, United States

Michael Maurer - Department of Chemistry, Tulane University, New Orleans, Louisiana 70118, United States

Xiaodong Zhang - Department of Chemistry, Tulane University, New Orleans, Louisiana 70118, United States

Stephen Sproules - WestCHEM, School of Chemistry, University of Glasgow, Glasgow G12 8QQ, United Kingdom; (1) orcid.org/0000-0003-3587-0375

Joel T. Mague - Department of Chemistry, Tulane University, New Orleans, Louisiana 70118, United States

Complete contact information is available at:

https://pubs.acs.org/10.1021/acs.inorgchem.1c01573

\section{Notes}

The authors declare no competing financial interest.

\section{ACKNOWLEDGMENTS}

The Louisiana Board of Regents [Grant LEQSF-(2002-03)ENH-TR-67] and the National Science Foundation (Grant MRI 1228232 and 0619770) are thanked for funding of Tulane University's X-ray crystallography and mass spectrometry instrumentation, and Tulane University is acknowledged for its ongoing assistance with operational costs for the X-ray diffraction facility. S.K. and J.P.D. gratefully acknowledge support for this project from the National Science Foundation (Grant CHE 1836569).

\section{REFERENCES}

(1) Arumugam, K.; Shaw, M. C.; Mague, J. T.; Bill, E.; Sproules, S.; Donahue, J. P. Long Range Spin Coupling: A TetraphosphineBridged Palladium Dimer. Inorg. Chem. 2011, 50, 2995-3002.

(2) Arumugam, K.; Selvachandran, M.; Obanda, A.; Shaw, M. C.; Chandrasekaran, P.; Caston Good, S. L.; Mague, J. T.; Sproules, S.; Donahue, J. P. Redox-Active Metallodithiolene Groups Separated by Insulating Tetraphosphinobenzene Spacers. Inorg. Chem. 2018, 57, 4023-4038.
(3) Zahavy, E.; Fox, M. A. An Os ${ }^{\mathrm{II}}-\mathrm{Ni}^{\mathrm{II}}-\mathrm{Pd}^{\mathrm{II}}$ Trimetallic Complex as an Electro-Switchable-Photoinduced-Electron-Transfer Device. Chem. - Eur. J. 1998, 4, 1647-1652.

(4) Chen, T.-H.; Popov, I.; Kaveevivitchai, W.; Miljanić, O. Š. MetalOrganic Frameworks: Rise of the Ligands. Chem. Mater. 2014, 26, $4322-4325$.

(5) Eisenhart, R. J.; Clouston, L. J.; Lu, C. C. Configuring Bonds between First-Row Transition Metals. Acc. Chem. Res. 2015, 48, 2885-2894.

(6) Boudier, A.; Breuil, P.-A. R.; Magna, L.; Olivier-Bourbigou, H.; Braunstein, P. Nickel(II) Complexes with Imino-Imidazole Chelating Ligands Bearing Pendant Donor Groups ( $\mathrm{SR}, \mathrm{OR}, \mathrm{NR}_{2}, \mathrm{PR}_{2}$ ) as Precatalysts in Ethylene Oligomerization. J. Organomet. Chem. 2012, $718,31-37$.

(7) Hartley, F. R.; Murray, S. G.; McAuliffe, C. A. Monomeric Complexes of Palladium(II) and Platinum(II) with a Series of OpenChain Tetrathioether Ligands Prepared from Complexes of Weak Donor Ligands. Inorg. Chem. 1979, 18, 1394-1397.

(8) Schrauzer, G. N.; Mayweg, V. P. Preparation, Reactions, and Structure of Bisdithio- $\alpha$-diketone Complexes of Nickel, Palladium and Platinum. J. Am. Chem. Soc. 1965, 87, 1483-1489.

(9) McFarlane, H. C. E.; McFarlane, W. Polyphosphorus Ligands V.* The Synthesis, Phosphorus-31 NMR Spectra and Conformations of the Polykis(diphenylphosphino) Benzenes $\left(\mathrm{Ph}_{2} \mathrm{P}\right)_{n} \mathrm{C}_{6} \mathrm{H}_{6-n}(n=1-$ 4). Polyhedron 1988, 7, 1875-1879.

(10) Genge, A R. J.; Levason, W.; Reid, G. Multinuclear NMR Studies of Diphosphine, Diphosphine-Dioxide and Diarsine Complexes of Tin(IV) Halides. Structures of $\left[\mathrm{SnI}_{4}\left\{0-\mathrm{C}_{6} \mathrm{H}_{4}\left(\mathrm{AsMe}_{2}\right)_{2}\right\}\right]$ and $\left[\mathrm{SnI}_{4}\left\{0-\mathrm{C}_{6} \mathrm{H}_{4}\left(\mathrm{P}(\mathrm{O}) \mathrm{Ph}_{2}\right)_{2}\right\}\right]$. Inorg. Chim. Acta 1999, 288, 142-149.

(11) Armarego, W. L. F.; Perrin, D. D. Purification of Laboratory Chemicals, 4th ed.; Butterworth-Heinemannn: Oxford, U.K., 2000.

(12) Arumugam, K.; Shaw, M. C.; Chandrasekaran, P.; Villagrán, D.; Gray, T. G.; Mague, J. T.; Donahue, J. P. Synthesis, Structures, and Properties of 1,2,4,5-Benzenetetrathiolate Linked Group 10 Metal Complexes. Inorg. Chem. 2009, 48, 10591-10607.

(13) Chandrasekaran, P.; Arumugam, K.; Jayarathne, U.; Pérez, L. M.; Mague, J. T.; Donahue, J. P. Synthesis, Structures, and Properties of Mixed Dithiolene-Carbonyl and Dithiolene-Phosphine Complexes of Tungsten. Inorg. Chem. 2009, 48, 2103-2113.

(14) Usón, R.; Vicente, J.; Oro, J. Maleonitriledithiolate Complexes of $\mathrm{Au}(\mathrm{III}), \mathrm{Au}(\mathrm{I}), \mathrm{Pd}(\mathrm{II})$, and $\mathrm{Pt}(\mathrm{II})$ Containing Neutral or Anionic Ligands. Inorg. Chim. Acta 1981, 52, 29-34.

(15) Cerrada, E.; Fernández, E. J.; Gimeno, M. C.; Laguna, A.; Laguna, M.; Terroba, R.; Villacampa, M. D. Synthesis of Dithiolate Gold(III) Complexes by Dithiolate Transfer Reactions. X-ray Structure of $\left[\mathrm{Au}\left(\mathrm{C}_{6} \mathrm{~F}_{5}\right)\left(\mathrm{S}_{2} \mathrm{C}_{6} \mathrm{H}_{4}\right)\left(\mathrm{PPh}_{3}\right)\right]$. J. Organomet. Chem. 1995, 492, 105-110.

(16) Cerrada, E.; Fernández, E. J.; Jones, P. G.; Laguna, A.; Laguna, M.; Terroba, R. Synthesis and Reactivity of Trinuclear Gold(III) Dithiolate Complexes. X-Ray Structure of $\left[\mathrm{Au}\left(\mathrm{C}_{6} \mathrm{~F}_{5}\right)\left(\mathrm{S}_{2} \mathrm{C}_{6} \mathrm{H}_{4}\right)\right]_{3}$ and $\left[\mathrm{Au}\left(\mathrm{C}_{6} \mathrm{~F}_{5}\right)\left(\mathrm{S}_{2} \mathrm{C}_{6} \mathrm{H}_{4}\right)\left(\mathrm{SC}_{6} \mathrm{H}_{4} \mathrm{SPPh}_{3}\right)\right]$. Organometallics 1995, 14, $5537-5543$

(17) Nomura, M.; Fourmigué, M. Dinuclear Cp* Cobalt Complexes of the 1,2,4,5-Benzenetetrathiolate Bischelating Ligand. Inorg. Chem. 2008, 47, 1301-1312.

(18) McGuire, J.; Miras, H. N.; Richards, E.; Sproules, S. Enabling Single Qubit Addressability in a Molecular Semiconductor Comprising Gold-Supported Organic Radicals. Chem. Sci. 2019, 10, 14831491.

(19) Shimizu, T.; Murakami, H.; Kobayashi, Y.; Iwata, K.; Kamigata, N. Synthesis, Structure, and Ring Conversion of 1,2-Dithiete and Related Compounds. J. Org. Chem. 1998, 63, 8192-8199.

(20) Obanda, A.; Martinez, K.; Schmehl, R. H.; Mague, J. T.; Rubtsov, I. V.; MacMillan, S. N.; Lancaster, K. M.; Sproules, S.; Donahue, J. P. Expanding the Scope of Ligand Substitution from $\left[\left(\mathrm{Ph}_{2} \mathrm{C}_{2} \mathrm{~S}_{2}\right)_{2} \mathrm{M}\right]\left(\mathrm{M}=\mathrm{Ni}^{2+}, \mathrm{Pd}^{2+}, \mathrm{Pt}^{2+}\right)$ to Afford New Heteroleptic Dithiolene Complexes. Inorg. Chem. 2017, 56, 10257-10267.

(21) Chandrasekaran, P.; Greene, A. F.; Lillich, K.; Capone, S.; Mague, J. T.; DeBeer, S.; Donahue, J. P. A Structural and 
Spectroscopic Investigation of Octahedral Platinum Bis(dithiolene)phosphine Complexes: Platinum Dithiolene Internal Redox Chemistry Induced by Phosphine Association. Inorg. Chem. 2014, 53, 9192-9205.

(22) Connick, W. B.; Gray, H. B. Photooxidation of Platinum(II) Diimine Dithiolates. J. Am. Chem. Soc. 1997, 119, 11620-11627.

(23) Brock, C. P.; Schweizer, W. B.; Dunitz, J. D. Internal Molecular Motion of Triphenylphosphine Oxide: Analysis of Atomic Displacement Parameters for Orthorhombic and Monoclinic Crystal Modifications at 100 and 150 K. J. Am. Chem. Soc. 1985, 107, 6964-6970.

(24) Davis, M. F.; Levason, W.; Reid, G.; Webster, M. Synthesis and Characterization of Tin(IV) Fluoride Complexes of Phosphine and Arsine Oxide Ligands. Polyhedron 2006, 25, 930-936.

(25) Codding, P. W.; Kerr, K. A. Triphenylphosphine Sulfide. Acta Crystallogr., Sect. B 1978, 34, 3785-3787.

(26) Clegg, W.; Edwards, A. J.; McFarlane, H. C. E.; McFarlane, W. 1,2,3,5-Tetrakis(diphenylthiophosphino)benzene: N.M.R. and X-ray Diffraction Studies of a Markedly Distorted Aromatic Molecule. Polyhedron 1998, 17, 3515-3518.

(27) Hursthouse, M. B.; Levason, W.; Ratnani, R.; Reid, G.; Stainer, H.; Webster, M. Synthesis, Spectroscopic and Structural Properties of an Unusual Series of Homoleptic Phosphine Oxide Complexes of the Alkaline Earth Dications. Polyhedron 2005, 24, 121-128.

(28) Schwab, M. M.; Himmel, D.; Kacprzak, S.; Radtke, V.; Kratzert, D.; Weis, P.; Wernet, M.; Peter, A.; Yassine, Z.; Schmitz, D.; Scheidt, E.-W.; Scherer, W.; Weber, S.; Feuerstein, W.; Breher, F.; Higelin, A.; Krossing, I. Synthesis, Characterizations, and Reactions of Truly Cationic $\mathrm{Ni}^{\mathrm{I}}$-Phosphine Complexes. Chem. - Eur. J. 2018, 24, 918927.

(29) Mayweg, V. P.; Schrauzer, G. N. Bis-adducts of Group VIII Metal Bisdithiobenzil Complexes with Phosphines. Chem. Commun. 1966, 640-641.

(30) Bowmaker, G. A.; Boyd, P. D. W.; Campbell, G. K. Electrochemical and ESR Studies of the Redox Reactions of Nickel(II), Palladium(II), and Platinum(II) Complexes of 1,2Diphenyl-1,2-ethenedithiolate(2-)-S,S'. Inorg. Chem. 1983, 22, $1208-1213$.

(31) Nomura, M.; Okuyama, R.; Fujita-Takayama, C.; Kajitani, M. New Synthetic Methods for $\eta^{5}$-Cyclopentadienyl Nickel(III) Dithiolene Complexes Derived from Nickelocene. Organometallics 2005, 24, 5110-5115.

(32) Connelly, N. G.; Geiger, W. E. Chemical Redox Agents for Organometallic Chemistry. Chem. Rev. 1996, 96, 877-910.

(33) McGuire, J.; Miras, H. N.; Donahue, J. P.; Richards, E.; Sproules, S. Ligand Radicals as Modular Organic Electron Spin Qubits. Chem. - Eur. J. 2018, 24, 17598-17605. 